\title{
Emergent Spatial Structure and Entanglement Localization in Floquet Conformal Field Theory
}

\author{
Ruihua Fan, ${ }^{1}$ Yingfei Gu๑, ${ }^{1}$ Ashvin Vishwanath, ${ }^{1}$ and Xueda Wen $\odot^{2}$ \\ ${ }^{1}$ Department of Physics, Harvard University, Cambridge, Massachusetts 02138, USA \\ ${ }^{2}$ Department of Physics, Massachusetts Institute of Technology, Cambridge, Massachusetts 02139, USA
}

(Received 18 September 2019; accepted 25 June 2020; published 14 August 2020)

\begin{abstract}
We study the energy and entanglement dynamics of $(1+1) \mathrm{D}$ conformal field theories (CFTs) under a Floquet drive with the sine-square deformed (SSD) Hamiltonian. Previous work has shown that this model supports both a nonheating and a heating phase. Here, we analytically establish several robust and "superuniversal" features of the heating phase which rely on conformal invariance but not on the details of the CFT involved. First, we show the energy density is concentrated in two peaks in real space, a chiral and an antichiral peak, which leads to an exponential growth in the total energy. The peak locations are set by fixed points of the Möbius transformation. Second, all of the quantum entanglement is shared between these two peaks. In each driving period, a number of Bell pairs are generated, with one member pumped to the chiral peak and the other member pumped to the antichiral peak. These Bell pairs are localized, accumulate at these two peaks, and can serve as a source of quantum entanglement. Third, in both the heating and nonheating phases, we find that the total energy is related to the half system entanglement entropy by a simple relation $E(t) \propto c \exp [(6 / c) S(t)]$ with $c$ being the central charge. In addition, we show that the nonheating phase, in which the energy and entanglement oscillate in time, is unstable to small fluctuations of the driving frequency in contrast to the heating phase. Finally, we point out an analogy to the periodically driven harmonic oscillator which allows us to understand global features of the phases and introduce a quasiparticle picture to explain the spatial structure, which can be generalized to setups beyond the SSD construction.
\end{abstract}

DOI: $10.1103 /$ PhysRevX.10.031036

\author{
Subject Areas: Condensed Matter Physics, \\ Particles and Fields, \\ Quantum Information
}

\section{INTRODUCTION}

Floquet driving sets up a new stage in the search for novel systems that may not have an equilibrium analog, such as Floquet topological phases [1-14] and time crystals [15-23]. It is also one of the simplest protocols to study nonequilibrium phenomena, such as localizationthermalization transitions, prethermalization, the dynamical Casimir effect, etc., [24-32]. However, exactly solving Floquet many-body systems is, in general, a formidable task. Usually, we have to resort to numerical methods limited to a small system size. This limitation makes an analytical understanding of Floquet dynamics extremely valuable. Conformal field theories provide an ideal platform for such a purpose $[33,34]$. In particular, for $(1+1) \mathrm{D}$ conformal field theories (CFTs), the conformal symmetry is enlarged to the full Virasoro symmetry, which makes the

Published by the American Physical Society under the terms of the Creative Commons Attribution 4.0 International license. Further distribution of this work must maintain attribution to the author(s) and the published article's title, journal citation, and DOI. calculation even more tractable $[35,36]$. In this paper, we focus on $(1+1)$ D CFTs. Generalization to other dimensions should be possible and is left to future work.

However, a CFT as a gapless many-body system is expected to be vulnerable to a generic driving. If we start from the ground state of the original Hamiltonian, then Floquet driving might lead it to an infinite temperature state easily. This thermalization process is an interesting problem but not the focus of this paper. Our goal is to explore what type of phenomena and structures can be engineered with a Floquet many-body system that may not be realized in a simple way with a static Hamiltonian. To avoid thermalization, we need to choose special protocols. In this paper, we use the Virasoro symmetry generators as our driving Hamiltonian so that we can take maximal advantage of the conformal symmetry to constrain the system. As one of the most canonical choices, we use the $\mathfrak{g l}(2, \mathbb{R})$ subalgebra, the exact meaning of which is discussed later. Although this choice may look a bit special, it is powerful enough to reveal some universal features of the problem that apply more generally. We also discuss one generalization of this simplest protocol.

We follow the setup used in Ref. [34], where the authors consider an open chain and implement the driving with the 
sine-square deformed Hamiltonian [37-47]. It is shown that if we start from the ground state and turn on the Floquet drive, we can identify a nonheating phase in the highfrequency driving regime and a heating phase in the lowfrequency driving regime by looking at the entanglement entropy growth. The fact that we have these two phases has an algebraic reason which can be understood by using a quantum mechanical model, as we discuss later.

The main part of this paper presents a more detailed study on what happens in the Floquet dynamics, paying special attention to the spatial structure that emerges that has not previously been discussed.

Let us summarize the main phenomena. In the heating phase, although the total energy and entanglement keep growing, the system does not evolve into a featureless state. We find that, in this phase, the system heats up in a very nonuniform way. The energy pumped in concentrates at two points, one of which has purely chiral excitations and the other one has only antichiral excitations. The entanglement entropy also comes from the entanglement between the excitations at these two points. Furthermore, the energy and entanglement entropy are related by a simple formula. All these features above are universal and depend only on the central charge of the CFT. In the nonheating phase, if we do a stroboscopic measurement, we can find that energy excitation will move back and forth in the system with the total energy and entanglement entropy oscillating in time.

Since a real experiment inevitably has noise, we are also interested in the question of how stable those phenomena are to noise. For example, we could start from an excited state or have local perturbation during evolution. Furthermore, the driving frequency could have a small fluctuation. By combining analytical and numerical analysis, we argue that the nonheating phase is delicate but all the reported features in the heating phase are quite robust to these perturbations. For the nonheating phase, an arbitrarily tiny noise in the driving frequency eventually heats the system. The dimensionless heating rate is proportional to $\alpha^{2}$, where $\alpha$ characterizes the magnitude of randomness.

The paper is organized as follows. In Sec. II, we briefly review the setup in Ref. [34], summarize the method of studying the evolution of operators, and see how to interpret the Hamiltonian by $\mathfrak{g l}(2, \mathbb{R})$ algebra. In particular, in Sec. II C, we discuss the phase diagram from a different angle using the mapping to a driven harmonic oscillator. In Sec. III, we present our main result of this paper on various features of the heating phase. We focus on how the energy is absorbed, how the entanglement is generated, and their relation. We also draw intuition from this special setup and make a few comments on what to expect for the case of more general initial conditions, boundary conditions, and Floquet drives. In Sec. IV, we analyze the stability of these phenomena against driving with random periods. In Sec. V, we introduce one generalization of the simplest case and study how the spatial structure of the energy and entanglement gets modified. Finally, in Sec. VI, we give some conclusions and the outlook.

\section{SETUP FOR A FLOQUET CFT}

In this section, in the interest of completeness, we review the setup and some results of prior work in Ref. [34] that are relevant to our discussion.

\section{A. Floquet driving}

We start with a $(1+1)$ D CFT with an open boundary condition. Let us denote its total length by $L$ and its central charge by $c$. We consider the following time-dependent Hamiltonian:

$$
H(t)= \begin{cases}H_{1}, & 0<t<T_{1}, \\ H_{0}, & T_{1}<t<T_{1}+T_{0},\end{cases}
$$

where $H_{0}$ is the ordinary Hamiltonian that can be written as an integral of energy density $T_{00}(x)$ along the real space as follows:

$$
H_{0}=\int_{0}^{L} d x T_{00}(x)
$$

$H_{1}$ is the so-called sine-square deformed Hamiltonian [37-48]:

$$
H_{1}=2 \int_{0}^{L} d x \sin ^{2}\left(\frac{\pi x}{L}\right) T_{00}(x) .
$$

For simplicity, the initial state $\left|\psi_{0}\right\rangle$ is chosen to be the ground state of $H_{0}$, i.e., $\left|\psi_{0}\right\rangle=|\mathrm{GS}\rangle$.

It is also useful to introduce the Floquet operator $F=$ $F_{0} F_{1}=e^{-i H_{0} T_{0}} e^{-i H_{1} T_{1}}$ to characterize the unitary evolution for a single cycle. In the stroboscopic measurement, the Floquet dynamics is determined by the state $|\psi(n T)\rangle=$ $F^{n}|\mathrm{GS}\rangle$. For example, the two-point function of local operators $O_{1}\left(x_{1}\right)$ and $O_{2}\left(x_{2}\right)$ after $n$ cycles is given by $\left\langle\psi(n T)\left|\mathcal{O}_{1}\left(x_{1}\right) \mathcal{O}_{2}\left(x_{2}\right)\right| \psi(n T)\right\rangle$. In the "Heisenberg" picture, the calculation amounts to determining the operator evolution $\mathcal{O}(x, n T)=F^{-n} \mathcal{O}(x) F^{n}$. For general Floquet drives, this problem is difficult. However, for the SSD Hamiltonian defined in Eq. (3), the operator evolution has a simple expression in terms of the Möbius transformation.

\section{B. Operator evolution and Möbius transformation}

In this section, we derive the explicit expressions for the operator evolution. It is convenient to work in Euclidean coordinates, and the Lorentzian correlator can be obtained by analytic continuation. We use three coordinates in this paper, denoted by

$$
w=\tau+i x, \quad z=e^{2 \pi w / L}, \quad \xi=e^{\pi w / L} .
$$




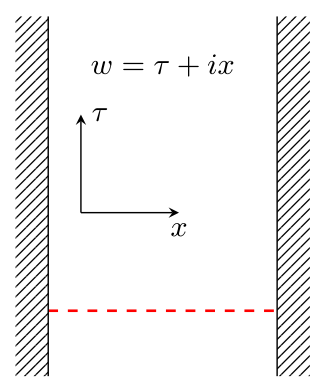

(a)

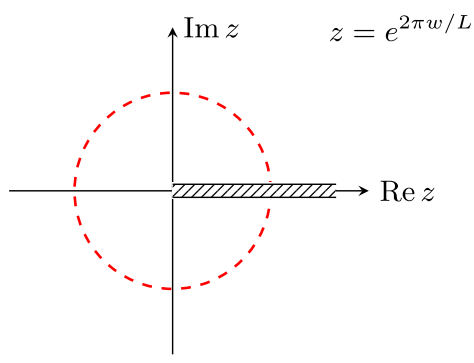

(b)

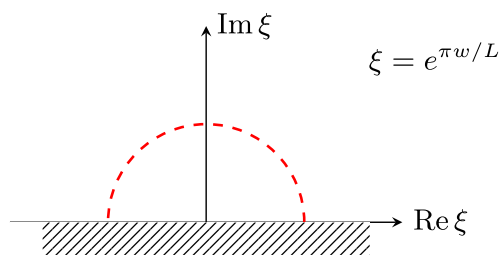

(c)

FIG. 1. Schematic plot of the three geometries. (a) Strip geometry where the $(1+1)$ D CFT with an open boundary is defined. (b) $z$ plane where we compute the operator evolution. (c) $\xi$ plane where we compute the operator expectation values.

They correspond to the stripe geometry, complex plane, and upper half-plane, respectively. See Fig. 1 for an illustration.

In the imaginary time, the Floquet operator is given by $F=e^{-\tau_{0} H_{0}} e^{-\tau_{1} H_{1}}$. Let us first check how the operator evolves after one cycle, namely,

$$
e^{H_{1} \tau_{1}} e^{H_{0} \tau_{0}} \mathcal{O}(w, \bar{w}) e^{-H_{0} \tau_{0}} e^{-H_{1} \tau_{1}} .
$$

Here, we assume $\mathcal{O}(w, \bar{w})$ to be a primary operator with the conformal dimension $(h, \bar{h})$. On the strip $w$, the algebraic relations between $H_{0,1}$ and $\mathcal{O}$ are complicated. It is easier to work in $z=e^{2 \pi w / L}$ coordinate instead, where $H_{0,1}$ are expressible as contour integrals of the stress tensor. More explicitly, we have

$$
\begin{aligned}
& H_{0}=\frac{2 \pi}{L} \int_{C} \frac{d z}{2 \pi i} z T(z)-(z \rightarrow \bar{z})-\frac{c \pi}{6 L}, \\
& H_{1}=\frac{2 \pi}{L} \int_{C} \frac{d z}{2 \pi i}\left(-\frac{1}{2}+z-\frac{z^{2}}{2}\right) T(z)-(z \rightarrow \bar{z})-\frac{c \pi}{6 L} .
\end{aligned}
$$

The term $-c \pi^{2} / 6 L$ comes from the Schwarzian derivative and does not affect the operator evolution. The contour $C$ is shown in Fig. 2(a). The subtlety is that $C$ is not closed due to the branch cut arising from the open boundary condition. The branch cut can be treated as follows. First, we use the Baker-Campbell-Hausdorff formula to expand Eq. (5) and write it as commutators. Each term can be depicted as a double contour integral shown in Fig. 2(b). The conformal boundary condition requires $T(z)=\bar{T}(\bar{z})$ right above and below the branch cut, respectively. Thus, we can attach two horizontal lines along with the branch cut [as the red horizontal lines in Fig. 2(b)] for free, since the contributions exactly cancel. After the above manipulations, the new contour can be deformed to enclose operator $O$ as shown in Fig. 2(c). Therefore, on the $z$ plane, the Floquet operator acts on the operators as if there is no branch cut.

As a consequence, the operator evolution that is driven by the stress tensor is determined by a two-step conformal transformation:

$$
\begin{aligned}
& e^{H_{1} \tau_{1}} e^{H_{0} \tau_{0}} \mathcal{O}(w, \bar{w}) e^{-H_{0} \tau_{0}} e^{-H_{1} \tau_{1}} \\
& =\left(\frac{\partial z}{\partial w}\right)^{h}\left(\frac{\partial \bar{z}}{\partial \bar{w}}\right)^{\bar{h}}\left(\frac{\partial z_{1}}{\partial z}\right)^{h}\left(\frac{\partial \bar{z}_{1}}{\partial \bar{z}}\right)^{\bar{h}} \mathcal{O}\left(z_{1}, \bar{z}_{1}\right),
\end{aligned}
$$

where $(\partial z / \partial w)$ corresponds to the transformation from the strip $(w)$ to the complex plane $(z)$ and $\left(\partial z_{1} / \partial z\right)$ is the transformation generated by the Floquet dynamics $F$.

To determine the map $z_{1}(z)$, we notice that, without the branch cut, $H_{0}$ and $H_{1}$ in Eq. (6) can be written as Virasoro generators $L_{0, \pm 1}$ and their antiholomorphic patterns as follows:

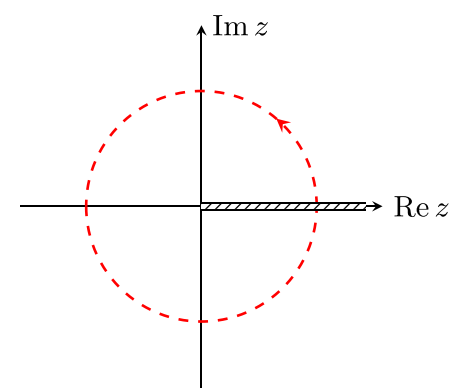

(a)

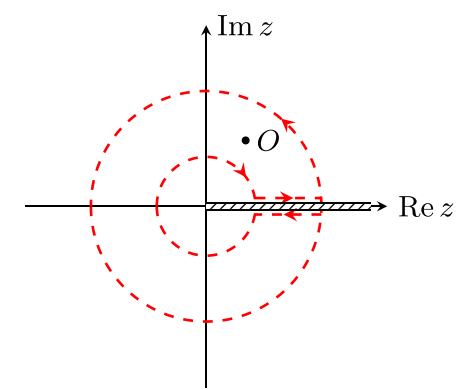

(b)

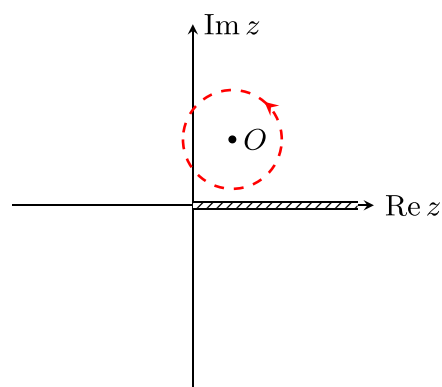

(c)

FIG. 2. (a) Integral contour for $H_{0}$ and $H_{1}$ on the $z$ coordinate. (b) Dashed circles are the integral contour for the commutator $\left[H_{0}, \mathcal{O}\right]$, $\left[H_{1}, \mathcal{O}\right]$; red horizontal lines are attached to circles to make it close. (c) The deformed contour. 


$$
\begin{aligned}
& \widetilde{H_{0}}=\frac{2 \pi}{L}\left(L_{0}+\bar{L}_{0}\right), \\
& \widetilde{H_{1}}=\frac{2 \pi}{L}\left(L_{0}-\frac{L_{-1}+L_{1}}{2}+\bar{L}_{0}-\frac{\bar{L}_{-1}+\bar{L}_{1}}{2}\right),
\end{aligned}
$$

where we use a tilde to emphasize that the identification works only for operator evolution. The generators $L_{0, \pm 1}$ form an $\mathfrak{g l}(2, \mathbb{R})$ algebra. Therefore, the corresponding Floquet operator $F$ generates a Möbius transformation on $z$, namely,

$$
z_{1}=f(z)=\frac{a z+b}{c z+d}, \quad\left(\begin{array}{ll}
a & b \\
c & d
\end{array}\right) \in \operatorname{SL}(2, \mathbb{R}) .
$$

The coefficients $a, b, c$, and $d$ are determined by the dimensionless driving periods $\tau_{0} / L$ and $\tau_{1} / L$ as follows:

$$
\begin{aligned}
& a=\left(1+\frac{\pi \tau_{1}}{L}\right) e^{\pi \tau_{0} / L}, \quad b=-\frac{\pi \tau_{1}}{L} e^{-\pi \tau_{0} / L}, \\
& c=\frac{\pi \tau_{1}}{L} e^{\pi \tau_{0} / L}, \quad d=\left(1-\frac{\pi \tau_{1}}{L}\right) e^{-\pi \tau_{0} / L} .
\end{aligned}
$$

More explicitly, the evolution induced by $H_{0}$ acts as a dilation on the $z$ plane; namely, $z$ goes to $\tilde{z}=e^{2 \pi \tau_{0} / L} z$, which explains the $e^{\pi \tau_{0} / L}$ factors. The evolution by $H_{1}$ is also a dilation but in a different coordinate $\chi$ [49].

For the Floquet problem, we would like to study the operator evolution for $n$ repeated cycles of Möbius transformations, namely, $z_{n}=f[f \ldots f(z)]$, and we denote it as

$$
z_{n}=f^{n}(z)=\frac{A z+B}{C z+D} .
$$

The successive application of Möbius transformation is better described using the fixed points $f(\gamma)=\gamma$ and the "rotations" $\eta$ relative to the fixed points

$$
\begin{aligned}
\gamma_{1} & =\frac{a-d-\sqrt{(a-d)^{2}+4 b c}}{2 c}, \\
\gamma_{2} & =\frac{a-d+\sqrt{(a-d)^{2}+4 b c}}{2 c}, \\
\eta & =\frac{c \gamma_{2}+d}{c \gamma_{1}+d} .
\end{aligned}
$$

With these new variables, Eq. (11) can be rearranged into the following form:

$$
\frac{z_{n}-\gamma_{1}}{z_{n}-\gamma_{2}}=\eta^{n} \frac{z-\gamma_{1}}{z-\gamma_{2}}
$$

For our physical application, $c=\left(\pi \tau_{1} / L\right) e^{\pi \tau_{0} / L}$ is nonzero, and there are, in general, three possible scenarios depending on the position of the fixed point.

(1) Elliptic class. - The quadratic equation $f(\gamma)=\gamma$ has two distinct roots that are conjugate to each other $\gamma_{1}=\gamma_{2}^{*}$, and the rotation parameter is determined by the following formula:

$$
\eta=\frac{c \gamma_{2}+d}{c \gamma_{1}+d} .
$$

In this case, $\eta$ is a pure phase, namely, $|\eta|=1$. See Fig. 3(a) for an illustration. The corresponding Möbius transformation $z_{n}=f^{n}(z)$ can be represented as an $\operatorname{SL}(2, \mathbb{R})$ matrix as follows"

$$
\left(\begin{array}{ll}
A & B \\
C & D
\end{array}\right)=\left(\begin{array}{cc}
\gamma_{1}-\eta^{n} \gamma_{2} & -\left(1-\eta^{n}\right) \gamma_{1} \gamma_{2} \\
1-\eta^{n} & -\left(\gamma_{2}-\eta^{n} \gamma_{1}\right)
\end{array}\right) .
$$

(2) Hyperbolic class. - The two distinct roots are purely real, and the parameter $\eta$ defined above is also a real number. See Fig. 3(c) for an illustration. The parameter $0<\eta<1$ represents the rescaling near the fixed points. The Möbius transformation matrix is in the same form as Eq. (15).

(3) Parabolic class.-Two roots are merged together: $\gamma_{1}=\gamma_{2}=\gamma$. Therefore, Eq. (13) does not apply. For this case, we introduce a new parameter $\beta=$ $[(a-d) / 2 c]$ such that

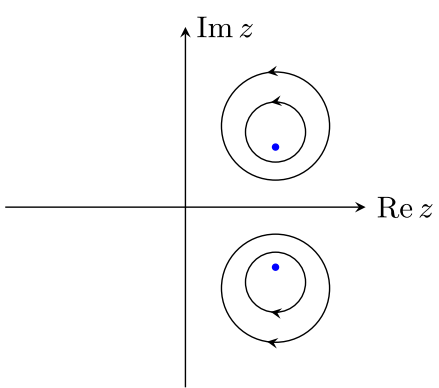

(a)

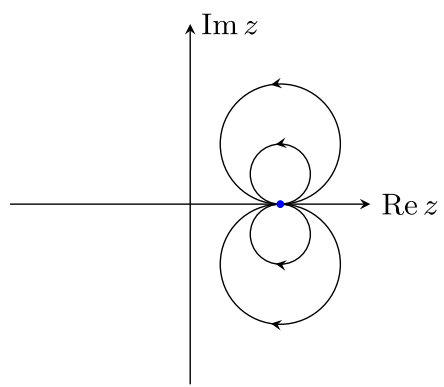

(b)

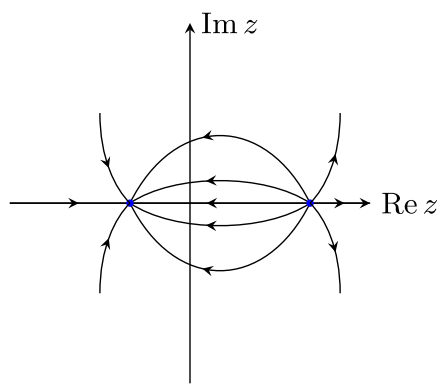

(c)

FIG. 3. Three classes of Möbius transformation: (a) elliptic, where two fixed points are two conjugate roots, and the orbits are circulating the fixed points; (b) parabolic, where the two fixed points coincide; (c) hyperbolic, where two fixed points are two real roots. 


$$
\frac{1}{z_{n}-\gamma}=\frac{1}{z-\gamma}+n \beta
$$

The corresponding transformation matrix is

$$
\left(\begin{array}{ll}
A & B \\
C & D
\end{array}\right)=\left(\begin{array}{cc}
1+n \beta \gamma & -n \beta \gamma^{2} \\
n \beta & 1-n \beta \gamma
\end{array}\right),
$$

which cannot be diagonalized. The parabolic class may be thought of as the marginal case of either the elliptic or hyperbolic class; see Fig. 3(b) for an illustration.

We remark here that the Möbius transformation also applies to quasiprimaries such as the stress tensor. In that case, although we obtain a Schwarzian derivative term when transforming between different geometries, the operator evolution driven by Möbius transformation on the complex plane is still determined by Eq. (7). More explicitly, the stress tensor on the strip after $n$-cycle driving becomes

$F^{-n} T(w) F^{n}=\left(\frac{\partial z}{\partial w}\right)^{2}\left(\frac{\partial z_{n}}{\partial z}\right)^{2} T\left(z_{n}\right)-\left(\frac{2 \pi}{L}\right)^{2} \frac{c}{24}$.

Finally, for the operator evolution in real (Lorentzian) time, we perform the analytic continuation $\tau_{0} \rightarrow i T_{0}$, $\tau_{1} \rightarrow i T_{1}$. In real time, a space-time position $(x, t)$ on the strip maps to $z=e^{i 2 \pi(x+t) / L}$ on the $z$ plane, which is always on the unit circle. Therefore, the operator evolution is geometrically related to the automorphism of a unit circle under the conformal mapping. Although the Möbius transformations after the analytic continuation generally belong to $\operatorname{SL}(2, \mathbb{C})$, the basic structure remains the same. (Naively, we may expect an additional class known as the loxodromic class to show up, where $\eta$ is a general complex number, not necessarily a phase or purely real. However, the physical parameters that appear in the Floquet setting do not fall into such a class.)

\section{Parametric oscillator (swing) analogy}

The last section explains the relation between the operator evolution and the Möbius transformation, which is further classified into three classes: elliptic, hyperbolic, and parabolic. The corresponding Floquet dynamics are also classified into the nonheating, heating, and critical classes, respectively, and the phase diagram was first obtained in Ref. [34]. For the reader's convenience, we reproduce the phase diagram in the Appendix A.

It is instructive and amusing to gain intuition into this classification in a more elementary setting with the same $\operatorname{SL}(2, \mathbb{R})$ structure. The example we use is the parametric oscillator with the following Hamiltonian [50,51]:

$$
H(t)=f(t) \frac{p^{2}}{2}+g(t) \frac{x^{2}}{2},
$$

where $f(t)=f(t+T)$ and $g(t)=g(t+T)$ are periodic functions. One familiar example is the Mathieu oscillator, which corresponds to $f(t)=1$ and $g(t)=g_{0}-2 g_{1} \cos (2 t)$. Classically, they are useful in explaining the motion of a playground swing; see Fig. 4(a) for a classical picture. Furthermore, the recognition of the $\operatorname{SL}(2, \mathbb{R})$ structure in the problem also has an interesting consequence in the ultracold quantum gases; e.g., see Refs. [52,53].

For the quadratic Hamiltonian, the Heisenberg operators $[x(t), p(t)]$ evolve under a $\operatorname{SL}(2, \mathbb{R})$ transformation that preserves the commutation relation $[x, p]=i,[54]$

$$
\begin{aligned}
\left(\begin{array}{l}
x(t) \\
p(t)
\end{array}\right) & =\left(\begin{array}{ll}
c_{11} & c_{12} \\
c_{21} & c_{22}
\end{array}\right)\left(\begin{array}{l}
x \\
p
\end{array}\right), \\
\left(\begin{array}{ll}
c_{11} & c_{12} \\
c_{21} & c_{22}
\end{array}\right) & \in \operatorname{SL}(2, \mathbb{R}) .
\end{aligned}
$$

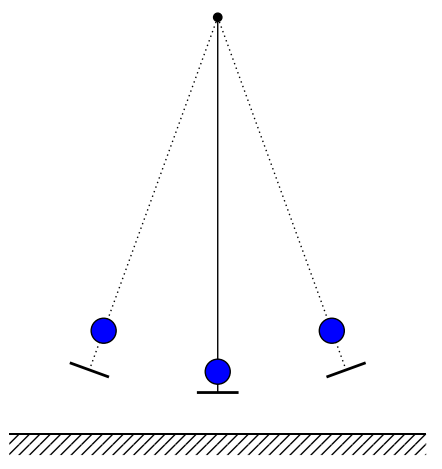

(a)

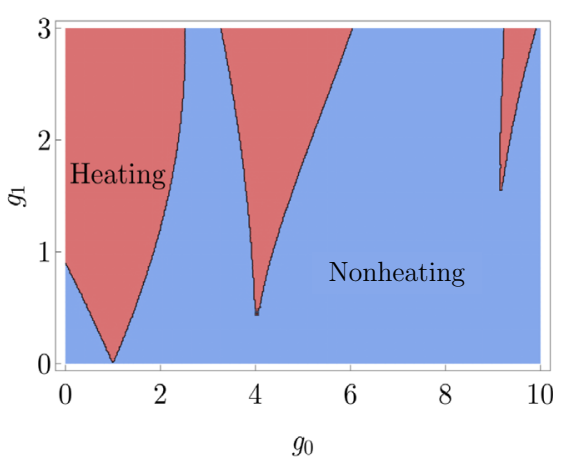

(b)

FIG. 4. (a) A simple example of a Mathieu oscillator is a child pumping a swing by periodically standing and squatting to increase the amplitude of the oscillation. The pump motion of a skilled child is typically at twice the frequency of the swing's oscillations which belongs to the heating phase. (b) Phase diagram of a Mathieu oscillator. In the red regime, energy keeps growing exponentially. In the blue regime, energy only oscillates. The black curve corresponds to the phase boundary, where energy grows quadratically. 
Therefore, the stroboscopic evolution of $(x, p)$ is represented by a $\operatorname{SL}(2, \mathbb{R})$ transformation $F_{(x, p)}$, whose classification determines the stroboscopic trajectory of $[x(n T)$, $p(n T)$ ]. More explicitly, to compare with the Möbius transformation used in Eq. (9), we treat $(x, p)$ as a point on the complex projective plane $\mathbb{C P}^{1}$ which can be more conveniently parametrized by $z=x / p$. Then, the $\operatorname{SL}(2, \mathbb{R})$ action on the point $(x, p)$ shown in Eq. (20) is equivalent to the Möbius transformation Eq. (9) and also has three classes. The fixed points $\gamma_{1,2}$ and the rotation angle $\eta$ of the Möbius transformation can be translated to the eigenvectors $v_{1,2}$ and the ratio of eigenvalues $\lambda_{1,2}$ of the $\operatorname{SL}(2, \mathbb{R})$ matrix, respectively. Their correspondence is given explicitly in Table I.

This translation explains the different dynamics. In the elliptic class, $(x, p)$ as a real vector keeps rotating only on the $x-p$ plane. The energy measured by $p^{2} / 2+x^{2} / 2$ just oscillates $E(n T) \sim \cos (n \theta+\varphi)$ with a period controlled by the angle $\theta$ of the eigenvalue. In the hyperbolic class, $F_{(x, p)}$ has two real right eigenvectors: $v_{1}$ with an eigenvalue $\lambda>1$ and $v_{2}$ with an eigenvalue $1 / \lambda<1$. Therefore, unless the initial condition $\left(x_{0}, p_{0}\right)$ is along $v_{2},[x(n T), p(n T)]$ flows to infinity along $v_{1}$ exponentially fast, which causes the energy to grow exponentially in the long-time limit:

$$
E(n T) \propto\left(p_{0} v_{2,1}-x_{0} v_{2,2}\right)^{2} \lambda^{2 n}, \quad n \gg 1 .
$$

In the parabolic class, $F_{(x, p)}$ has only one right eigenvector $v$ with eigenvalue 1 and, thus, becomes singular. To determine the dynamics, we can look at the Jordan normal form of $F_{(x, p)}$ [55]. Unless the initial condition $\left(x_{0}, p_{0}\right)$ is along $v,[x(n T), p(n T)]$ flows to infinity linearly, which causes the energy to grow quadratically:

$$
E(n T) \propto\left(p_{0} v_{1}-x_{0} v_{2}\right)^{2} n^{2} .
$$

As a concrete example, the Mathieu oscillator introduced at the beginning of this section can support all of the three different dynamics, and its phase diagram is presented in Fig. 4.

The Floquet CFT studied in the current paper is richer than its oscillator analog. In particular, the $(1+1) \mathrm{D}$ CFT has locality in space, which leads to features in the energy density and entanglement that are the focus of the following sections.

\section{ENERGY AND ENTANGLEMENT}

Energy and entanglement are the most straightforward and fundamental diagnostics of states evolving under Floquet driving. Fortunately, both can be studied in $(1+1) \mathrm{D}$ CFT analytically using the operator evolution method we discuss. In this section, we present the stroboscopic measurement of the energy and entanglement under Floquet driving. We also provide a semiclassical picture of the phenomenon and point out an interesting relation between energy and entanglement.

\section{A. Energy density and total energy}

The energy of a state under the Floquet evolution can be measured by the expectation value of the stress tensor $T_{00}=T+\bar{T}$, whose time evolution can be obtained by Eq. (18). The $n$ dependence of the energy arises from the first term. To compute $\left\langle G\left|T\left(z_{n}\right)\right| G\right\rangle$, we need to perform another conformal transformation to the upper half-plane via $\xi=\sqrt{z}$. This mapping generates a Schwarzian derivative

$$
\frac{c}{12} \operatorname{Sch}(\xi, z)=\frac{c}{12}\left[\frac{\xi^{\prime \prime \prime}(z)}{\xi^{\prime}(z)}-\frac{3}{2}\left(\frac{\xi^{\prime \prime}(z)}{\xi^{\prime}(z)}\right)^{2}\right]=\frac{c}{32 z^{2}}
$$

and leaves a second term $\langle G|T(\xi)| G\rangle$. On the one hand, the Ward identity and scale invariance constrain $\langle G|T(\xi)| G\rangle \propto$ $1 / \xi^{2}$. On the other hand, it is invariant under the horizontal translation. Therefore, this term has to vanish, and all the contribution comes from the Schwarzian term, namely,

$$
\left\langle\mathrm{GS}\left|F^{-n} T(w) F^{n}\right| \mathrm{GS}\right\rangle=\left(\frac{\partial z}{\partial w}\right)^{2}\left(\frac{\partial z_{n}}{\partial z}\right)^{2} \frac{c}{32 z_{n}^{2}},
$$

where $w=\tau+i x$ is the complex coordinate for the stress tensor $T$ on the strip, $z_{n}$ is the coordinate on the $z$ plane after $n$-cycle driving, and $c$ is the central charge. The initial value is subtracted and is ignored in the rest of the discussion in this section. After analytic continuation $\tau_{0} \rightarrow i T_{0}, \tau_{1} \rightarrow i T_{1}$, the expectation value of $T$ has the following form:

$\langle T\rangle(x, t=n T)=\left(\frac{2 \pi}{L}\right)^{2} \frac{c}{32} \frac{(A D-B C)^{2} z^{2}}{(A z+B)^{2}(C z+D)^{2}}$,

with $A, B, C$, and $D$ depending on $T_{0} / L, T_{1} / L$, and $n$ through the prescription described in Sec. II B. Replacing $z$ with $\bar{z}$ gives us the expectation value of $\bar{T}$.

TABLE I. The dictionary between the Möbius transformation and the $\mathrm{SL}_{2}$ matrix for the parametric oscillator.

\begin{tabular}{lccccc}
\hline \hline & \multicolumn{2}{c}{ Möbius transformation } & & \multicolumn{2}{c}{$\operatorname{SL}(2, \mathbb{R})$ matrix $F_{(x, p)}$} \\
\cline { 2 - 3 } Classification & Fixed points & & & Eigenvectors & Eigenvalues \\
\hline Elliptic & $\gamma_{1}, \gamma_{2} \in \mathbb{C}$ & & & $v_{1}, v_{2} \in \mathbb{C}$ & $\lambda_{1}=\lambda_{2}=e^{i \theta / 2}$ \\
Hyperbolic & $\gamma_{1}, \gamma_{2} \in \mathbb{R}$ & $0<\eta<1$ & & $v_{1}, v_{2} \in \mathbb{R}$ & $\lambda_{1}=1 / \lambda_{2}<1$ \\
Parabolic & $\gamma_{1}=\gamma_{2} \in \mathbb{R}$ & 1 & & $v_{1}=v_{2} \in \mathbb{R}$ & $\lambda_{1}=\lambda_{2}=1$ \\
\hline \hline
\end{tabular}




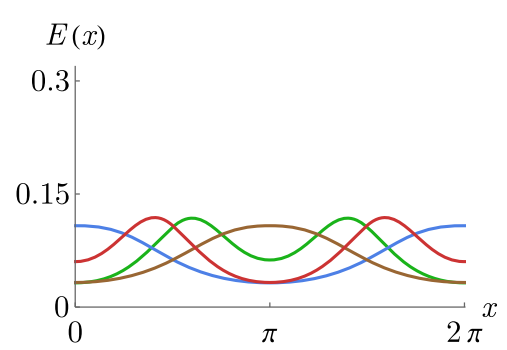

(a)

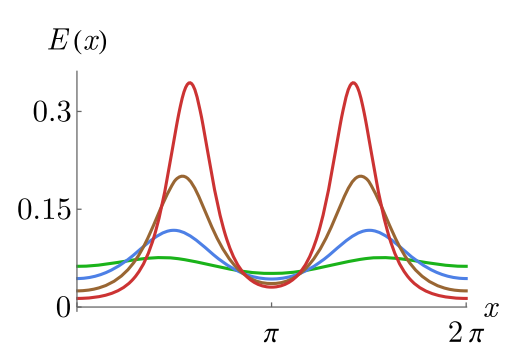

(b)

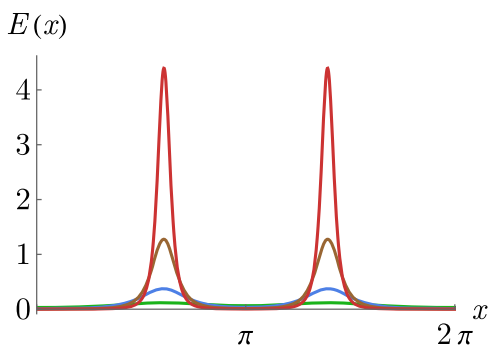

(c)

FIG. 5. The evolution of energy density profile in different phases. In the plot, we choose the system size $L=2 \pi$ and the central charge $c=1$. Different colors mean different times. The green, blue, brown, and red curves correspond to $t=T, 2 T, 3 T, 4 T$, respectively. (a) $T_{0}=0.5 \mathrm{~L}$ and $T_{1}=0.1 \mathrm{~L}$, which is in the nonheating phase. The energy density oscillates only (b) $T_{0}=0.9 \mathrm{~L}$, and $T_{1}$ is tuned to make the system right at the phase boundary. The position of the peaks in the plot are not given by $\gamma$, because we are not at the late-time regime. One can check that, as we increase $n$, the peaks move toward $\log \gamma$. (c) $T_{0}=0.9 L$ and $T_{1}=0.1 L$, which is in the heating phase. We can clearly see the formation and growth of two peaks.

These formulas allow us to look at the evolution of energy density directly, which is found to have different behaviors in different phases, as shown in Fig. 5. In the nonheating phase, the energy density just fluctuates without a definite period. In the heating phase, the energy density quickly develops two peaks, which grow with time vary fast. The positions of the energy peaks are determined by the unstable fixed points of the Möbius transformation, i.e., $e^{2 \pi i x_{\text {peak }} / L}=\gamma_{2}$ or $\gamma_{2}^{*}$. At the phase boundary, there are also two energy peaks but growing much slower.

These phenomena can be understood from the perspective of the fixed points of Möbius transformation. The $n$ dependence enters Eq. (24) through two parts: (a) the Schwarzian term $c / 32 z_{n}^{2}$, which has a constant magnitude due to the fact that $\left|z_{n}\right|=1$ in real time; (b) the rescaling factor $\left(\partial z_{n} / \partial z\right)^{2}$, whose different behaviors in three phases explain the feature shown in Fig. 5.

(1) Nonheating phase.-The two fixed points sit on different sides of the unit circle. In our convention, $\gamma_{1}$ is inside the unit circle, and $\gamma_{2}$ is outside the unit circle, as depicted in Fig. 6(a). Since $z_{n}$ is constrained on the unit circle, it cannot flow to either of them but just keeps rotating around them. That is the reason that energy density fluctuates in this phase. Since the rotation angle $\eta$, defined by Eq. (14), is not a rational phase, these fluctuations do not have a definite period.

(2) Heating phase.-Both fixed points are now on the unit circle. In our convention, $\gamma_{1}$ is a stable fixed point, and $\gamma_{2}$ is an unstable fixed point. For the chiral stress tensor, when $z=\gamma_{2}$, although $z_{n}$ does not change, the rescaling factor $\left(\partial z_{n} / \partial z\right)^{2}=\eta^{-2 n}$ grows exponentially with $n$. For the antichiral stress tensor, the same thing happens at $\bar{z}=\gamma_{2}$. Therefore, we observe two energy peaks at two symmetric positions. On the other hand, for a generic position $z, \bar{z} \neq \gamma_{2}, z_{n}$ flows to the stable fixed point, and the rescaling factor $\left(\partial z_{n} / \partial z\right)^{2}$ decreases exponentially with $n$. Therefore, the stress tensor shrinks, making the two peaks sharper and sharper. In a lattice system, the energy peaks are also observed and consistent with the CFT prediction in the short time. In the late time, they saturate and oscillate due to having only a finite number of degrees of freedom, as detailed in Appendix D.

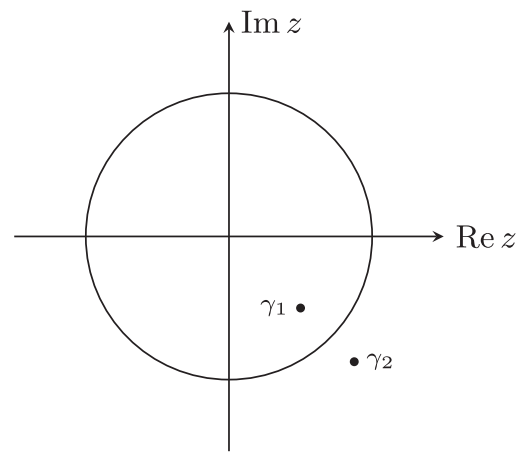

(a)

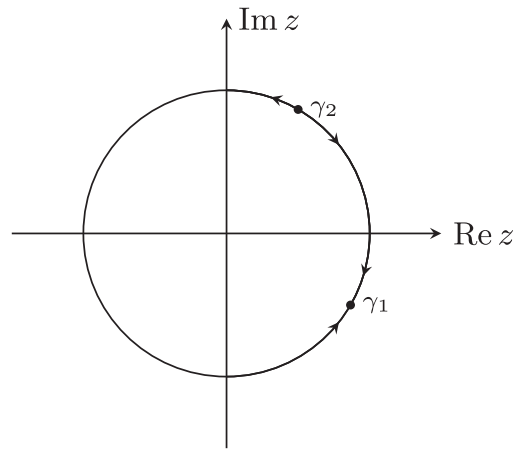

(b)

FIG. 6. Fixed point distribution in real time. (a) In the nonheating phase, one fixed point is inside the unit circle, while the other one is outside. (b) In the heating phase, both fixed points are on the unit circle. One is attractive, and the other is repulsive. 
(3) Critical line.-The two fixed points merge to $\gamma$ on the unit circle, which is a marginal case. One can show that the maximal value of the rescaling factor keeps growing but in a power-law fashion, which explains the slowly growing peaks. The position for the maximum gradually moves to the position corresponding to $\gamma$.

Note that the phenomena here do not rely on the initial state, as long as it is not a common eigenstate of $H_{0}$ and $H_{1}$. For example, we may consider a generic initial state $|\phi\rangle$ with the expectation value of stress tensor $\langle\phi|T(z)| \phi\rangle=$ $\mathcal{E}_{\phi}(z)$, then Eq. (24) generalizes to

$$
\left\langle\phi\left|F^{-n} T(w) F^{n}\right| \phi\right\rangle=\left(\frac{\partial z}{\partial w}\right)^{2}\left(\frac{\partial z_{n}}{\partial z}\right)^{2} \mathcal{E}_{\phi}(z),
$$

and the discussions above still hold. In this scenario, the boundary condition is also irrelevant, since the operator evolution discussed in Sec. II B is independent of the choice of boundary conditions [56].

The idea of relating the fixed points to the heating and nonheating phenomena also applies to more general setups. For example, we can use $H_{0}$ and $H_{2}=2 \int_{0}^{L} d x \sin ^{2}(2 \pi x /$ $L) T_{t t}(x)$ to generate the Floquet dynamics. $H_{2}$ is related to $L_{ \pm 2}$, and, thus, the operator evolution is still a conformal transformation but with four fixed points. When none of the fixed points are on the unit circle, the system is in the nonheating phase without energy peaks. In a certain parameter regime, there are two unstable fixed points locating on the unit circle, which implies heating dynamics and correspondingly four growing energy peaks. Furthermore, we can define a Hamiltonian by a generic deformation $H=\int_{0}^{L} d x f(x) T_{t t}(x)$. As long as $f(x)$ is a smooth real function and has a Fourier decomposition, $H$ can be represented as a linear combination of Virasoro generators, and the operator evolution can be written as a conformal transformation [57]. If $f(x)=\sin ^{2}(k \pi x / L), k \geq 1$, the conformal mapping is essentially the same as what we discuss here, which supports a nonheating and a heating phase. However, for a generic $f(x)$, the determination of fixed points and the corresponding dynamics is a hard problem, which we leave for a future study.

Besides the energy density, we can also look at the total energy

$$
E(t)=\int_{0}^{L} \frac{d x}{2 \pi}(\langle T\rangle+\langle\bar{T}\rangle) .
$$

For stroboscopic measurement, we can plug in Eq. (24) and have

$$
E(t=n T)=\frac{2 \pi}{L} \frac{c}{16} \frac{A D+B C}{A D-B C} .
$$

In either the nonheating or heating phase, the Möbius transformation has two fixed points, and we need to use Eq. (15) to get

$$
\begin{aligned}
E(t=n T) & =\frac{2 \pi}{L} \frac{c}{16} \frac{A D+B C}{A D-B C} \\
& =\frac{2 \pi}{L} \frac{c}{16} \frac{-2 \gamma_{1} \gamma_{2}+\left(\gamma_{1}+\gamma_{2}\right)^{2} \eta^{n}-2 \gamma_{1} \gamma_{2} \eta^{2 n}}{\eta^{n}\left(\gamma_{1}-\gamma_{2}\right)^{2}} .
\end{aligned}
$$

For the nonheating phase, since $\eta$ is a pure phase, the total energy oscillates with time. Generally, $\eta$ is a nonrational phase factor; thus, we do not expect any periodicity. Since the energy is oscillating, we cannot talk about the long-time behavior itself but the average

$$
\begin{aligned}
\bar{E}_{\text {nonheating }} & :=\lim _{n \rightarrow \infty} \frac{1}{n} \sum_{k=1}^{n} E(t=k T) \\
& =\frac{2 \pi}{L} \frac{c}{16}\left(\frac{\gamma_{1}+\gamma_{2}}{\gamma_{1}-\gamma_{2}}\right)^{2}
\end{aligned}
$$

which is a finite number. For the heating phase, $0<\eta<1$ and $\eta^{n}$ becomes exponentially small at the late-time regime. Therefore, to the leading order, we can drop the $\eta^{n}$ and $\eta^{2 n}$ terms in the numerator and find the energy grows exponentially:

$$
E_{\text {heating }}(t=n T) \approx \frac{2 \pi}{L} \frac{c}{16} \frac{-2 \gamma_{1} \gamma_{2}}{\left(\gamma_{1}-\gamma_{2}\right)^{2}} \eta^{-n},
$$

for $n \gg 1$.

When the system is at the boundary between the nonheating and heating phases, the two fixed points merge together, and we need to use Eq. (17). Noting that $\beta \gamma$ is pure imaginary, the total energy can be written as

$$
E_{\text {phase boundary }}(t=n T)=\frac{2 \pi}{L} \frac{c}{16}\left(1+2|\beta \gamma|^{2} n^{2}\right) .
$$

The total energy grows quadratically in $n$.

This long-time asymptotics of the total energy provides a direct diagnostic of the different phases. The oscillation and exponential and quadratic growth behavior match the simple picture obtained in the driven harmonic oscillators, as shown in Sec. II C. In particular, noticing that $\eta=\lambda^{-2}$, the heating rate in Eqs. (21) and (31) are exactly the same, because the growth behavior depends only on the underlying algebra and not its detailed realization.

\section{B. Entanglement pattern in the heating phase}

Besides the total energy, the entanglement entropy of the left and right half system also has different behaviors in different phases, which is shown in Ref. [34]. Here, we focus on the spatial structure of entanglement that has not previously been discussed. In particular, we examine the heating phase and discuss the relation between the energy peaks observed above and the entanglement. 
Given a pure state $|\Psi\rangle$, the reduced density matrix of a subsystem $A$ is defined by the partial trace $\rho_{A}=$ $\operatorname{Tr}_{\bar{A}}|\Psi\rangle\langle\Psi|$, and its von Neumann entanglement entropy is given as $S_{A}=-\operatorname{Tr} \rho \log \rho$. In our setting, we consider a time-dependent state $|\psi(n T)\rangle=F^{n}|\mathrm{GS}\rangle$ that evolves under the Floquet driving and study the corresponding entanglement entropy $S_{A}(n T)$ as a function of driving cycle $n$.

For a subsystem $A=[0, x]$ starting from the left end and ending at position $x \in(0, L)$, we plot the results in Fig. 7 and keep the details of the calculation in Appendix B. The entanglement entropy has a background value from the initial state. As time increases, the curve quickly develops two kinks, the positions of which exactly coincide with the energy peaks. Only the curve between the two kinks grows with time, while the curve outside does not. This difference implies that only when the subsystem includes one of the energy peaks does the entanglement grow with time. If the subsystem includes either none or both peaks, the entanglement remains at its background value and does not grow at all.

This statement can be further verified by studying the entanglement of the subsystem $A^{\prime}$ with ending points $x_{1}, x_{2} \in(0, L)$. We fix $x_{2}$ to sit between the two energy peaks and study how the long-time behavior of entanglement growth depends on the choice of $x_{1}$. Without loss of generality, we assume the chiral energy peak is on the left and the antichiral peak is on the right in the following discussion. In general, there are three different choices of $x_{1}$.

(1) $0<x_{1}<x_{C}$. - In this case, the subsystem $A^{\prime}$ includes only the chiral peak, as depicted in Fig. 8(a). The entanglement entropy is

$$
S_{A^{\prime}}\left(x_{1}, x_{2}, t\right)=-\frac{c}{6} n \log \eta+(\text { nonuniversal }),
$$

where the first term grows linearly with time (i.e., the driving cycle $n$ ), which is consistent with the result

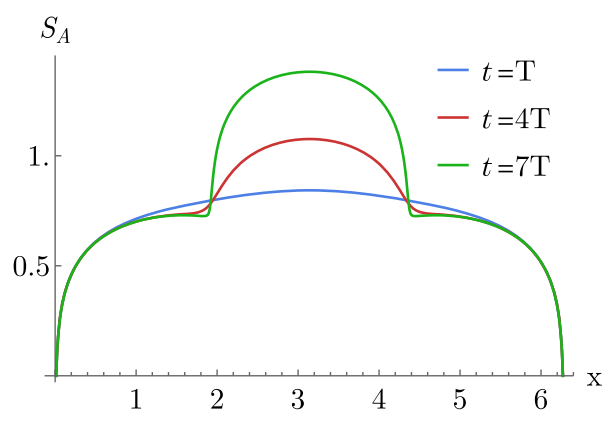

FIG. 7. Entanglement entropy of the subsystem $A=[0, x]$ as a function of entanglement cut $x$. Different colors represent different times. As time increases, the curve develops two kinks. Only curves between the two kinks grow with time. The positions of the kinks are the same as the positions of the energy peaks shown in Fig. 5. In this plot, we choose the system size $L=2 \pi$, and $T_{0}=0.9 L$ and $T_{1}=0.1 L$. in Ref. [34]. As long as $x_{1}<x_{C}$, the slope depends only on the central charge and the characteristic constant $\eta$ but not on the positions of entanglement cuts. This behavior is universal and does not depend on the operator content. The nonuniversal terms are subleading in the $n \gg 1$ limit.

(2) $x_{C}<x_{1}, x_{2}<x_{A}$.-In this case, the subsystem is between the chiral and the antichiral peak, as depicted in Fig. 8(b). To the leading order, one can show that it saturates to an $\mathcal{O}(1)$ value, which depends on the operator content and position of insertion. The exact value is not relevant, but the most important is that the entanglement entropy does not have interesting time dependence in the longtime limit.

(3) $x_{A}<x_{1}$. - In this case, the subsystem $A^{\prime}$ includes only the antichiral peak, as depicted in Fig. 8(c). The entanglement entropy grows linearly as in Eq. (33). We also provide the lattice calculation to further check these statements. The results can be found in Appendix D.

Using the results above, we can also infer bipartite mutual information between the two energy peaks. Let us choose two disjoint regions $X$ and $Y$, with $X$ and $Y$ including only the left and right peak, respectively. We call their complement as $Z$, which is composed of three disjoint regions $Z_{1}, Z_{2}$, and $Z_{3}$, located on the left of the chiral peak, between the two peaks, and on the right of the antichiral peak, respectively. Since we are studying a pure state, the mutual information between $X$ and $Y$ is

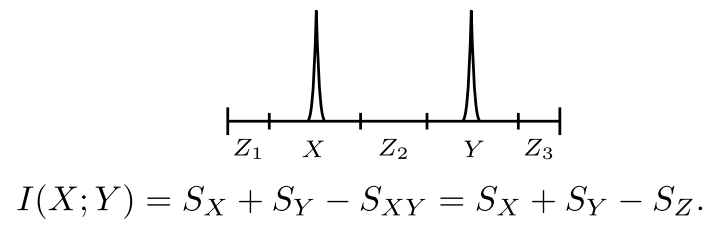

Following the prescriptions in Appendix B, the calculation of $S_{X Y}$ requires a four-point function of twist operators in the boundary CFT which is, in general, unknown. Instead, we can use the subadditivity of entanglement to bound $S_{Z}$ :

$$
0 \leq S_{Z} \leq S_{Z_{1}}+S_{Z_{2}}+S_{Z_{3}} \sim \mathcal{O}(1)
$$

where in the last step we use the fact that none of $S_{Z_{j}}$, $j=1,2,3$, grows with time and saturates to an $\mathcal{O}(1)$ value. Therefore, $S_{Z}$ itself can be only the $\mathcal{O}(1)$ value and does not make an important contribution to the time dependence of the mutual information. On the other hand, since each of $X$ and $Y$ includes one peak, $S_{X}$ and $S_{Y}$ grow linearly with time as shown in Eq. (33). Thus, the mutual information also linearly grows with time:

$$
I(X ; Y)=-\frac{c}{3} n \log \eta+(\text { nonuniversal }),
$$




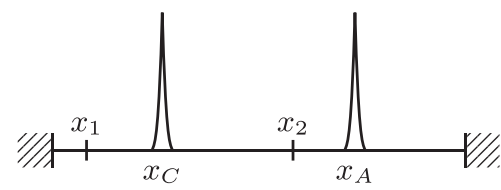

(a)

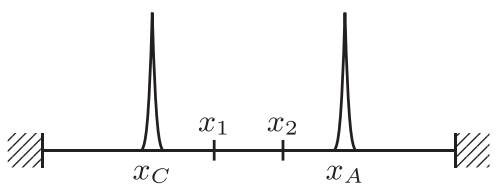

(b)

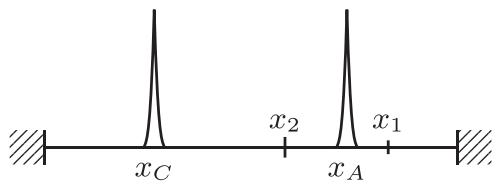

(c)

FIG. 8. Entanglement cuts for different cases. In (a) and (c), the subsystem includes only one energy peak. In (b), the subsystem does not include any energy peak. (a) $x_{1}<x_{C}$ (b) $x_{C}<x_{1}<x_{A}$ (c) $x_{1}>x_{A}$.

where the nonuniversal terms are subleading in the $n \gg 1$ limit.

All of the results above provide strong evidence that the state prepared by this Floquet driving contains only bipartite entanglement. We can think of the entanglement pattern as being described by many EPR pairs accumulating at the two peaks; i.e., one member of the pair is at one peak, and the other member of the pair is at the other peak. In each Floquet cycle, there are $(c / 3) \log _{2}(1 / \eta)$ pairs created.

This result suggests a quasiparticle picture which is developed in the next section and helps us understand the phenomena outlined by the calculations.

\section{The quasiparticle picture}

In this section, we provide a quasiparticle picture to understand the formation of the peaks and the entanglement pattern similar to the discussions in Calabrese and Cardy [58,59]. It is not surprising that such a quasiparticle picture exists, since our analysis above should apply to any $(1+1) \mathrm{D}$ CFT, including the one realized by a $(1+1) \mathrm{D}$ massless free fermion. What is interesting is that the predictions from the quasiparticle picture agree quantitatively with the CFT calculations.

From the quasiparticle picture, in each Floquet driving cycle, when we suddenly change the Hamiltonian, we expect that there will be quasiparticle excitations emitting from different points. The pairs of particles moving to the left and right from a given point are highly entangled. For example, at the beginning of each cycle, we change $H_{0}$ to $H_{1}$, which creates EPR pairs in the system. Then, they move together with all other quasiparticles that have been created in previous cycles with velocity $v(x)=2 \sin ^{2}(\pi x / L)$. The velocity is determined by the sin-square envelope we define in Eq. (3). In the second part of each driving cycle, the quasiparticles are governed by $H_{0}$, and the velocity now changes to $v(x)=1$. These are sketched in Fig. 9.

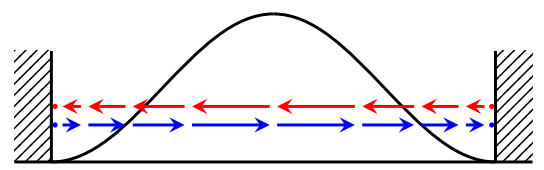

(a)
Therefore, we can determine the distance that a quasiparticle travels in one cycle by the following formula:

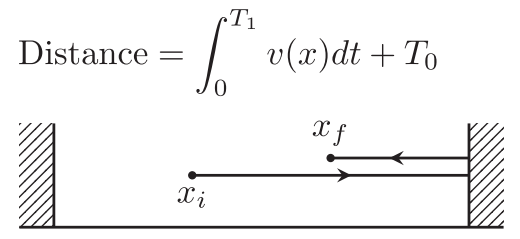

This distance depends on $T_{0}, T_{1}$, and the initial position $x_{i}$ of the quasiparticle, thus uniquely determining the final position $x_{f}$ of the quasiparticle. For the plot in Eq. (37), we assume the quasiparticle bounces back once from the boundary [60]. In general, the number of bounces is $n$ if $(n-1) L<T_{0}<n L$, where $n$ is a positive integer. One can find that $T_{1}$ does not come in, because under $H_{1}$ the quasiparticles never reach the boundary due to the vanishing of velocity at the boundary [61].

With this quasiparticle picture, we can determine the positions of the two energy peaks as observed in Fig. 5. Recall that in the long-time limit $n \gg 1$, after each driving cycle, the positions of the two energy peaks stay the same. There are only two possibilities as follows (without loss of generality, let us focus on the chiral energy peak and track its position $x_{C}$ ).

(1) If the number of bounces $n$ is odd, then the chiral energy peak becomes an antichiral energy peak due to the bounces at the boundary. To keep the positions of the chiral and antichiral energy peaks the same, we have to do the switching: $x_{C} \leftrightarrow x_{A}$. That is, we have $x_{i}=x_{C}$ and $x_{f}=x_{A}$ in Eq. (37).

(2) If the number of bounces $n$ is even, then the chiral energy peak is still chiral after each driving cycle. Then, one has $x_{i}=x_{f}=x_{C}$.

The tracking of the antichiral energy peak can be analyzed in the same way. Noting that the two energy peaks are

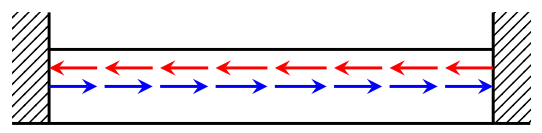

(b)

FIG. 9. Quasiparticle picture: In both (a) and (b), black lines are the cartoon profile for the Hamiltonians, and the red (blue) arrows are the corresponding velocities for the left- (right-) moving quasiparticles. (a) SSD Hamiltonian and (b) Uniform Hamiltonian. 
symmetric about $x=L / 2$, i.e., $x_{A}=L-x_{C}$, we can determine the positions of the two energy peaks by the "quantization" condition

$$
\int_{0}^{T_{1}} v(x) d t+T_{0}=n L, \quad n \in \mathbb{Z} .
$$

Evaluating the above equation explicitly, one can find that $x_{C}$ and $x_{A}$ are determined by the following equation:

$$
\frac{2 \pi T_{1}}{L}=\cot \frac{\pi\left(x^{*}+T_{0}-n L\right)}{L}-\cot \frac{\pi x^{*}}{L},
$$

with $x_{C}=x^{*}$ and $x_{A}=L-x^{*}$. One can check that the solution $x^{*}$ in Eq. (39) matches the repulsive fixed point $\gamma_{2}=e^{2 \pi i x^{*} / L}$ of the Möbius transformation in Eq. (12) exactly.

This quasiparticle picture turns out to be useful. On the one hand, it gives us a semiclassical explanation for the formation of the peaks. In the heating phase, the system keeps absorbing energy by creating many EPR pairs. Because of the fixed point solution in the semiclassical motion, those EPR pairs accumulate at the fixed point, with the chiral part staying at $x_{C}=x^{*}$ and the antichiral part staying at $x_{A}=L-x^{*}$. If we keep track of what happens within one cycle, we find that the particles at the two peaks switch their position after one cycle if the number of bounces $n$ is odd but stay the same if $n$ is even. In the nonheating phase, the system does not absorb much energy, and there is no fixed point in the equation of motion. Therefore, we observe only energy oscillation.

On the other hand, it also provides us insight into the growth of entanglement entropy. The EPR pairs generated not only carry energy but also share entanglement. Therefore, as the system absorbs energy, the entanglement also grows. Based on this semiclassical picture, it is not hard to conjecture that all the entanglement is shared by the two peaks. Since the energy and entanglement are carried by the same objects, it is also natural to expect some relationship between them. In the following section, we derive such a relation.

\section{Energy-entanglement relation}

As we said before, since we have this interpretation that the energy and entanglement are all carried by those EPR pairs at the two peaks, it is natural to ask whether there is any relation between them. The result for entanglement entropy always contains a divergent nonuniversal piece due to the absence of a UV cutoff in a field theory, which is absent on the energy side. Therefore, in the following, we compare only their universal time dependence and dispense with the nonuniversal part.

First, let us look at the results for the heating phase. By comparing Eq. (31) with Eq. (33), we can find the following equation:

$$
E_{\text {heating }}(t) \propto c \exp \left(\frac{6}{c} S(t)\right)
$$

which relates the total energy growth to the entanglement growth for the chiral or antichiral peaks. We use proportion instead of equality because we keep only the universal information and drop all the other nonuniversal details.

Then, let us see whether this relation also holds in the nonheating phase and the critical case. Because we already know that the entanglement comes from the two peaks, it is sufficient and technically easier if we just use the result for the left half and right half entanglement, as computed in Ref. [34]. Since they use a different notation, we reproduce their results in Appendix B using our notations so that readers can compare with the total energy more conveniently. For the nonheating phase, the entanglement entropy keeps oscillating as $\# \eta^{n}$ around a nonzero average value, which matches this result. For the critical case, the entanglement entropy grows logarithmically in time $S \approx(c / 3) \log n$, which also matches this result.

Several remarks follow below. This equation contains only the central charge and, thus, is true for any CFT. Generically, we would not expect such a universal relation. It holds here only because the states prepared are related by a conformal transformation that makes the universal relation possible [62]. It is also, to some extent, reminiscent of the Cardy formula in an equilibrium CFT, which says energy is proportional to the square of the entropy. However, what we find here is that the energy is the exponential of the entropy, and thus much larger than the entropy, which suggests the state we prepare is far from the equilibrium state.

\section{EFFECT OF RANDOMNESS IN NONHEATING PHASE}

In a real experiment, local perturbations and imperfections of the pulse sequences are inevitable. In this section, we discuss the effect of having some small randomness on the driving period $T_{0}$ and $T_{1}$; i.e., in each cycle, $T_{0}$ and $T_{1}$ are independently drawn from a certain distribution, and the final results are obtained after doing a "disorder" average. Here are some detailed explanations of the protocol.

(1) The driving time for each cycle is given by

$$
T_{0}=\bar{T}_{0}+\delta T_{0}, \quad T_{1}=\bar{T}_{1}+\delta T_{1},
$$

where $\delta T_{0}$ and $\delta T_{1}$ denote the deviation from the (constant) mean values $\bar{T}_{0}$ and $\bar{T}_{1}$. For simplicity, we consider the case that $\delta T_{0}$ and $\delta T_{1}$ are uniformly distributed in the following domain:

$$
\delta T_{0}, \delta T_{1} \in\left[-\frac{\alpha L}{2}, \frac{\alpha L}{2}\right],
$$


where $\alpha$ characterizes the magnitude of randomness. $L$ is the total length of the system, which is the fundamental timescale of the system.

(2) Given a sequence of randomized driving time, the operator $\mathcal{O}$ on the $z$ plane under $n$-cycle imaginary time evolution is given by the familiar formula

$$
\left(\frac{\partial z_{n}}{\partial z}\right)^{h}\left(\frac{\partial \bar{z}_{n}}{\partial \bar{z}}\right)^{\bar{h}} \mathcal{O}\left(z_{n}, \bar{z}_{n}\right)
$$

The derivative terms are calculated using the chain rule:

$$
\frac{\partial z_{n}}{\partial z}=\frac{\partial z_{n}}{\partial z_{n-1}} \cdot \frac{\partial z_{n-1}}{\partial z_{n-2}} \cdots \frac{\partial z_{2}}{\partial z_{1}} \cdot \frac{\partial z_{1}}{\partial z}
$$

where [cf. Eqs. (9) and (10)]

$$
z_{i}=f\left(z_{i-1}\right)=\frac{a z_{i}+b}{c z_{i}+d}, \quad\left(\begin{array}{ll}
a & b \\
c & d
\end{array}\right) \in \operatorname{SL}(2, \mathbb{R}) .
$$

The difference comparing to the previous sections is that the parameters $a, b, c$, and $d$ here are determined by the randomized driving cycles; in particular, the terms in the chain rule formula are independent.

We comment that, in principle, for the real-time evolution, we need to analytically continue for each cycle in order to keep track of the trajectories of $z_{n}$ and $\bar{z}_{n}$ to determine the branch cut crossing. The disorder average is done after the analytic continuation. Here, we consider only the stress tensor and the entanglement entropy of the left (right) half system. Both quantities are free of the branch cut issue, and we can safely perform the analytic continuation.

\section{A. Energy and entanglement growth}

In this section, we present numerical results for the energy and entanglement growth affected by randomness. We focus on the nonheating phase and briefly comment on the heating phase.

The first result is presented in Fig. 10, where we choose parameters $\bar{T}_{0}, \bar{T}_{1}$, and $\alpha \ll 1$ such that each individual sample $\left(T_{0}, T_{1}\right)$ belongs to the nonheating phase, namely, corresponds to an elliptic Möbius transformation. With the randomized protocol, we find that the total energy of the system grows with time (even for $\alpha \ll 1$ ). Asymptotically, it grows exponentially with $n$ as numerically verified in Fig. 10(a). At the same time, the entanglement entropy grows linearly with $n$, as shown in Fig. 10(b). That is to say, the nonheating phase disappears immediately for arbitrarily weak randomness, and we are left with only the heating phase. The total energy and entanglement entropy grow in the same way as what we find for the heating phase without any randomness. As a side note, if we implement the random driving setup for a Mathieu oscillator, small randomness also leads the energy to grow exponentially (see Appendix $\mathrm{C}$ for details), which suggests that the phenomenon here might be a generic feature of the $\operatorname{SL}(2, \mathbb{R})$ algebra and not special to our CFT setting.

Then, it is desirable to ask how the heating rate is related to the magnitude of randomness $\alpha$. We study this problem based on the entanglement entropy, as follows. With randomness, there are four dimensionless parameters in the calculation of entanglement: $l_{A} / L, \bar{T}_{0} / L, \bar{T}_{1} / L$, and $\alpha$. Recalling that $S(n)$ also depends on the total length $L$ of

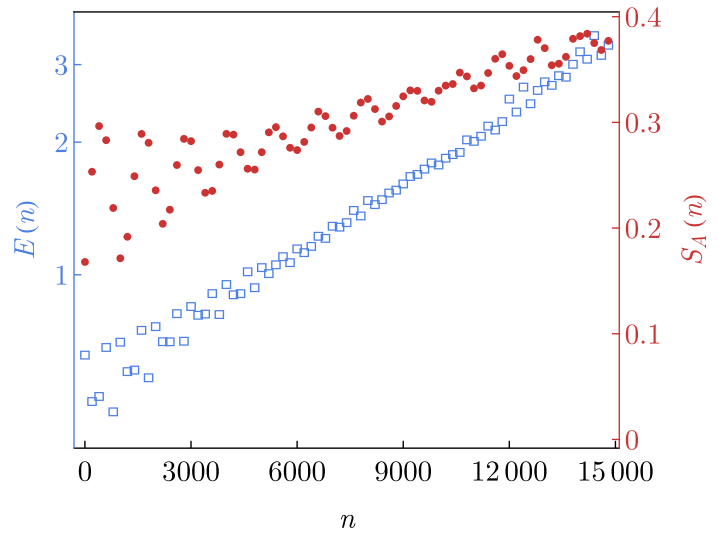

(a)

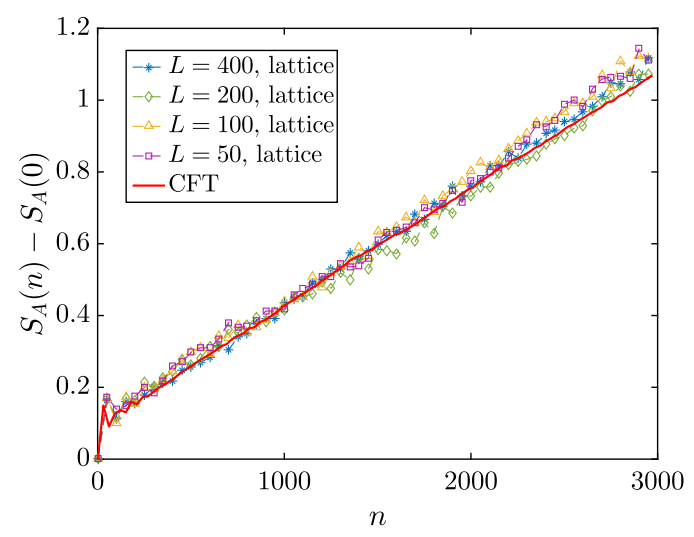

(b)

FIG. 10. The total energy and entanglement entropy of the subsystem $A=[0, L / 2]$ with random driving. (a) We choose $L=2 \pi$, and $\bar{T}_{0}=\bar{T}_{1}=\bar{T}, \bar{T} / L=0.1$, and $\alpha=0.01 . n$ is the number of driving cycles. Each data point is calculated by doing a random average 1000 times. The energy (blue hollow squares) grows with time exponentially. The entanglement entropy (red dots) for the left half system grows with time linearly. (b) Entanglement entropy evolution for subsystem $A=[0, L / 2]$ with different $L$. The lattice calculation is done with complex free fermions and is averaged over $N_{\text {sample }}=200$, with $L=50,100,200$, and 400, respectively. The randomness is chosen as $\bar{T}_{0}=\bar{T}_{1}=\bar{T}, \bar{T} / L=0.03$, and $\alpha=0.06$. 


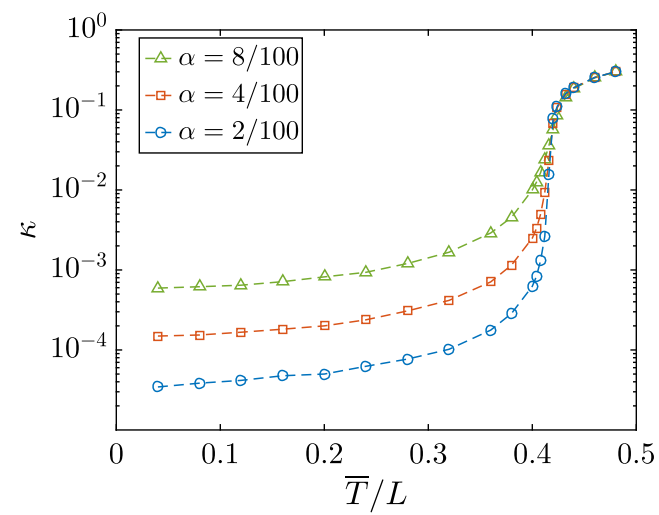

(a)

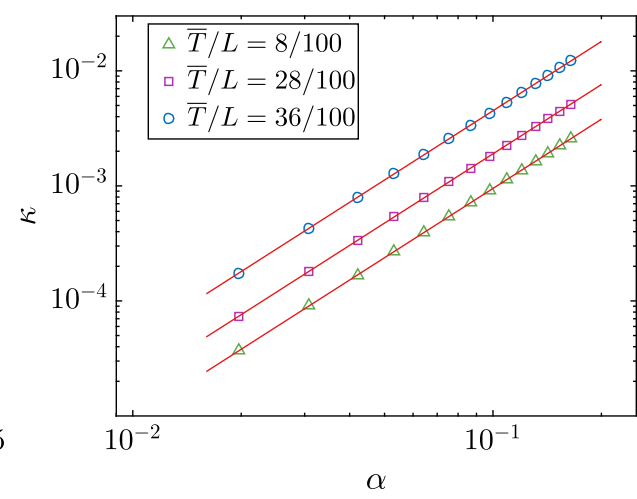

(b)

FIG. 11. (a) The slope $\kappa$ of entanglement entropy growth as a function of $\bar{T} / L$, for different magnitudes of randomness $\alpha$. Note that the phase transition from nonheating to heating phases with no randomness $(\alpha=0)$ happens at $T^{*} / L \simeq 0.416$. (b) The slope $\kappa$ of entanglement entropy growth as a function of $\alpha$, for different $\bar{T}$. The red solid lines are fittings with $\kappa \propto \alpha^{2}$. Each $\kappa$ is extracted from the CFT calculation of $S_{A}(n)$ by averaging over $N_{\text {sample }}=1000$.

the system through its initial value, we consider that the quantity $S_{A}(n)-S_{A}(0)$ depends only on the dimensionless ratios we introduce above [63]. Let us introduce the heating rate $\kappa$ and write the entropy growth as

$$
S_{A}(n)-S_{A}(0)=\kappa \cdot n+\text { const. }
$$

For simplicity, we consider the choice of $T_{0}$ and $T_{1}$ in Eq. (41) with $\bar{T}_{0}=\bar{T}_{1}=\bar{T}$. Then, for $A=[0, L / 2], \kappa$ depends only on two dimensionless parameters, i.e., $\bar{T} / L$ and $\alpha$.

As shown in Fig. 11(a), we study $\kappa$ as a function of $\bar{T} / L$ with different $\alpha$. There are several interesting features: (i) Fixing $\alpha$, as $\bar{T}$ increases from $\bar{T} / L=0, \kappa$ increases accordingly. In particular, $\kappa$ grows the fastest near the phase transition $T^{*} / L \simeq 0.416$ (note that the phase transition is defined for the case with no randomness). This result indicates that the system is more sensitive to the randomness near the phase transition. (ii) Fixing $\bar{T}<T^{*}$, one can find that $\kappa$ increases with the randomness $\alpha$. That is, with larger fluctuations in the driving periods, the system is heated up more easily. (iii) For $\bar{T}>T^{*}, \kappa$ collapse to the same curve, indicating that the heating phase (defined before adding noise) is robust under the effect of fluctuations in the driving periods.

With the analysis above, now we are interested in how $\kappa$ depends on the magnitude of randomness $\alpha$ for a fixed $\bar{T}$ with $\bar{T}<T^{*}$, which corresponds to the nonheating phase (before adding randomness). As shown in Fig. 11(b), it is found that $\kappa$ depends on $\alpha$ in the following way:

$$
\kappa \propto \alpha^{2}
$$

where the coefficient $\kappa / \alpha^{2}$ depends on $\bar{T}$, as can be seen in Fig. 11(b). With this observation, one can alternatively plot $\kappa / \alpha^{2}$ as a function of $\bar{T}$. It is found that $\kappa / \alpha^{2}$ for $\bar{T}<T^{*}$ depends only on $\bar{T}$, with the concrete value $0.1-1$.

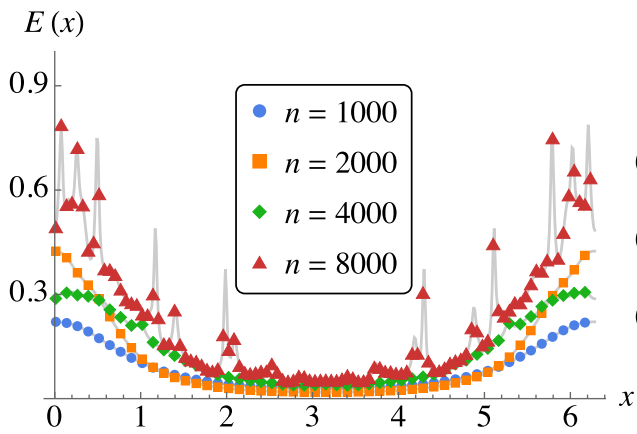

(a)

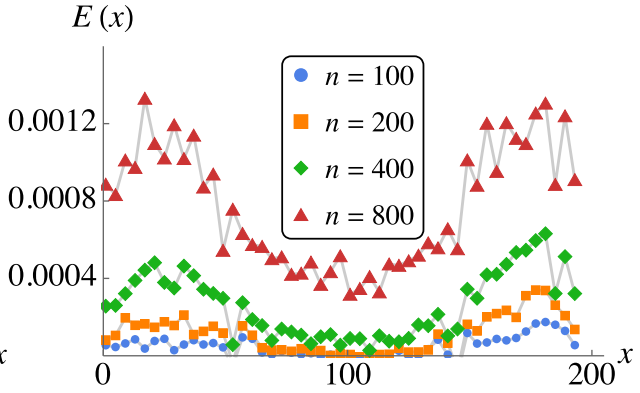

(b)

FIG. 12. Energy density for random driving. (a) CFT result on weak randomness. We choose $L=2 \pi$, and $\bar{T}_{0}=\bar{T}_{1}=\bar{T}, \bar{T} / L=0.1$, and $\alpha=0.01$. Each curve is calculated by doing a random average 1000 times. (b) Lattice calculation on strong randomness. We simulate a complex free fermion on the lattice with $L=200, \bar{T}_{0}=\bar{T}_{1}=\bar{T}, \bar{T} / L=0.3, \alpha=0.03$, and half filling. Each curve is calculated by doing a random average 300 times. In both calculations, we find the energy density peaks on the boundary. 


\section{B. Energy density distribution}

After determining how the randomness in the driving periods $T_{0}$ and $T_{1}$ affects the phase diagram, let us look at how it changes the energy density distribution.

If we start from the nonheating phase regime and add randomness, we find that the energy density peaks at the two ends of the system, as shown in Fig. 12. This phenomenon can be understood from the semiclassical quasiparticle picture. Without randomness, the quasiparticles are created and moved in the system coherently. After adding randomness, those motions become irregular. However, since the group velocity of the quasiparticles is smaller near the boundary, accordingly we have a higher probability to see more quasiparticles there.

However, if we start from the heating phase regime, the total energy keeps growing exponentially with time and the energy peaks do not disappear for moderate randomness, as shown in Fig. 13. We can first drive the system with a fixed period and let the energy peaks form. Then, we turn on sufficiently weak randomness. Now, the energy peaks are not moved back perfectly but with a small discrepancy. As a result, the energy peaks are smeared a little bit but still there. Therefore, just as the time crystal with many-body localization [20-22], all the features for the heating phase we find here are also robust even though we slightly perturb it away from the fine-tuning (randomness-free) point.

Before we close this section, we emphasize that, in our CFT calculation of the energy and entanglement evolution in the presence of randomness (see Fig. 10), the average is performed numerically. It is desirable to derive an analytic result, for example, for the heating rate $\kappa$ in Fig. 11. We leave this problem for future study.

\section{GENERALIZATION TO OTHER SUBALGEBRA}

Appealing to the quasiparticle picture, our setup has a natural generalization, i.e., replacing the SSD with other arbitrary envelope functions $\int_{0}^{L} d x f(x) T_{00}(x)$. In this section, we consider a specific one, where $f(x)$ involves only a single Fourier component:

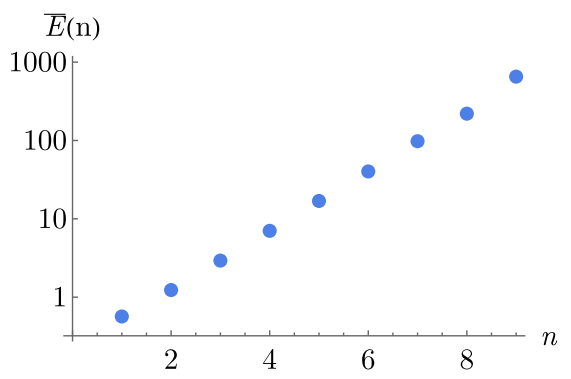

(a)

$$
\begin{aligned}
H_{q} & =\int_{0}^{L} d x\left(1-\cos q \frac{2 \pi x}{L}\right) T_{00}(x) \\
& =2 \int_{0}^{L} d x \sin ^{2}\left(q \frac{\pi x}{L}\right) T_{00}(x), \quad q=2,3,4, \ldots
\end{aligned}
$$

A periodic boundary condition is used in this section for the sake of simplicity, but most phenomena shown below are qualitatively the same for the open boundary condition [64]. Before detailing the results, let us first explain some intuition for this generalization from the algebraic viewpoint and the quasiparticle picture.

To understand it from the algebraic viewpoint, we rewrite $H_{q}$ in terms of the Virasoro generators:

$$
H_{q}=\frac{2 \pi}{L}\left(L_{0}-\frac{L_{-q}+L_{q}}{2}+\bar{L}_{0}-\frac{\bar{L}_{-q}+\bar{L}_{q}}{2}\right),
$$

in which only $L_{0, \pm q}$ (and $\bar{L}_{0, \pm q}$ ) appear. They again form a $\mathfrak{I} \mathfrak{l}(2, \mathbb{R})$ subalgebra [65] and, therefore, follow the same classification scheme we discuss before, i.e., nonheating, heating phase, and the critical line.

From the quasiparticle picture, the SSD Hamiltonian $H_{1}$ (with a periodic boundary condition) introduces one zero point at the identified edge for the spatial profile of the velocity $v(x)$, while $H_{q}$ puts $q$ zeros and arranges them with a equal spacing $L / q$. Let us denote the region $[(m-1) L / q]<x<(m L / q)$ as $R_{m}, m=1,2, \ldots, q$. For each $R_{m}$, the system can be treated as if being governed by $H_{0}$ and $H_{1}$. It implies that there will be two energy peaks in each $R_{m}$, and the only difference is that the quasiparticles can move to nearby interval $R_{m \pm 1}$ after one cycle.

Next, we elaborate the details of the above intuitions with a focus on the energy and entanglement patterns in the heating phase.

\section{A. Operator evolution}

In this section, we derive the formula for the operator evolution by working in Euclidean time and performing the

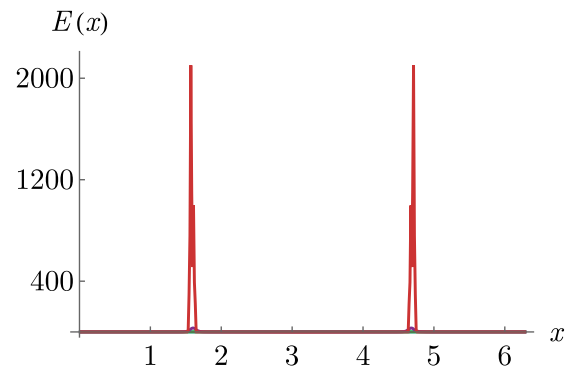

(b)

FIG. 13. Dynamics in the heating phase regime with small randomness. We choose $L=2 \pi, T_{0}=0.9 L, T_{1}=0.15 L$, and $\alpha=0.01$. Each data point is averaged over 100 times. (a) Averaged total energy still grows with time exponentially. (b) The energy density distribution is the same as that without any randomness, except that the peaks are smeared out a little. 
analytical continuation at the end. The whole procedure is similar to that shown in Sec. II B.

To calculate the operator evolution after a single-cycle driving, we need a conformal mapping from the cylinder to a more convenient geometry. The above observation about each region $R_{m}$ leads to the following conformal transformation:

$$
z=e^{q(2 \pi w / L)}=e^{2 \pi w / l}, \quad w=\tau+i x,
$$

where $l=L / q$ is the length of each region $R_{n}$. For a fixed $\tau, z$ winds the origin $q$ times as $x$ increases from 0 to $L$, which implies that $z$ describes a $q$-sheet Riemann surface with the $q$-fold branch cut being $[0,+\infty)$. Let us introduce $H_{0(q)}\left[R_{m}\right]$ as the part of the total Hamiltonian $H_{0(q)}$ supported on the region $R_{m}$, and then its expression in the $z$ coordinate is

$$
\begin{aligned}
H_{0}\left[R_{m}\right]= & \frac{2 \pi}{l} \int_{C, n} \frac{d z}{2 \pi i} z T(z)-(z \rightarrow \bar{z})-\frac{c \pi}{6 l}, \\
H_{q}\left[R_{m}\right]= & \frac{2 \pi}{l} \int_{C, n} \frac{d z}{2 \pi i}\left(-\frac{1}{2}+z-\frac{z^{2}}{2}\right) T(z) \\
& -(z \rightarrow \bar{z})-\frac{c \pi}{6 l},
\end{aligned}
$$

which is locally the same as Eq. (6) except that the total system size $L$ is replaced with the subregion size $l$ and $m$ is introduced as the Riemann sheet label. As a result, the operator evolution on this $q$-sheet Riemann surface is also described by an $\operatorname{SL}(2, \mathbb{R})$ transformation:

$$
z_{1}=\frac{a z+b}{c z+d}, \quad\left(\begin{array}{ll}
a & b \\
c & d
\end{array}\right) \in \operatorname{SL}(2, \mathbb{R}),
$$

with the dimensionless coefficients being

$$
\begin{aligned}
& a=\left(1+\frac{\pi \tau_{1}}{l}\right) e^{\pi \tau_{0} / l}, \quad b=-\frac{\pi \tau_{1}}{l} e^{-\pi \tau_{0} / l}, \\
& c=\frac{\pi \tau_{1}}{l} e^{\pi \tau_{0} / l}, \quad d=\left(1-\frac{\pi \tau_{1}}{l}\right) e^{-\pi \tau_{0} / l} .
\end{aligned}
$$

It has the same form as that for the simplest case derived in Sec. II B with $L$ replaced by $l$. The formula for multiple repeated cycles is the composition of the above transformation and is denoted by the same Eq. (11) with $l$ used in the definition of parameters.

Therefore, the operator evolution in this generalized protocol has the same classification as the previously discussed case $(q=1)$, and the phase diagram of the dynamics is identical to Fig. 16 as long as the total system size $L$ is replaced with the subregion size $l$. On the other hand, the introduction of $q$ Riemann sheets enriches the spatial structure of the operator evolution; e.g., the fixed points on one sheet are duplicated to all the sheets, and, therefore, the entanglement pattern is enriched as we see shortly.

\section{B. Energy density}

According to the above discussion, the time-evolved stress tensor is

$F^{-n} T(w) F^{n}=\left(\frac{\partial z}{\partial w}\right)^{2}\left(\frac{\partial z_{n}}{\partial z}\right)^{2} T\left(z_{n}\right)+\frac{c}{12} \operatorname{Sch}(z, w)$.

Evaluated on the ground state of $H_{0}$, we obtain

$$
\begin{aligned}
\langle T(x, t=n T)\rangle= & \frac{\pi^{2} c}{6 L^{2}} \cdot\left(q^{2}-1\right) \\
& \cdot \frac{(A D-B C)^{2} z^{2}}{(A z+B)^{2}(C z+D)^{2}}-\frac{q^{2} \pi^{2} c}{6 L^{2}} .
\end{aligned}
$$

Note $L=q l$. Here, $A, B, C$, and $D$ also follow the prescription in Sec. II B with $L$ replaced by $l$. For $\langle\bar{T}(x, t=n T)\rangle$, one simply replaces $z$ with $\bar{z}$ in Eq. (56). The total energy $E(t=n T)=\int_{0}^{L}(d x / 2 \pi)(T+\bar{T})$ grows as

$E(t=n T)=-\frac{q^{2} \pi c}{6 L}+\frac{\pi c}{6 L} \cdot\left(q^{2}-1\right) \cdot \frac{A D+B C}{A D-B C}$.

Several remarks follow.

(1) For $q=1$, one can find that $\langle T(x, t=n T)\rangle=$ $\langle\bar{T}(x, t=n T)\rangle=-\left(\pi^{2} c / 6 l^{2}\right)=-\left(q^{2} \pi^{2} c / 6 L^{2}\right)$, which contains only the Casimir energy of the ground state. This result is because, for $q=1$, the ground states of $H_{0}$ and $H_{q=1}$ are the same, and, therefore, there is no nontrivial time evolution as mentioned in Reference [64].

(2) For $q>1$, the feature of energy growth in each region $R_{m}$ is the same as those as discussed in Sec. III [66]. When the system is in the heating phase, we observe two energy peaks in each of the $q$ regions, one from $T$ and the other from $\bar{T}$. An example with $q=4$ is shown in Fig. 14; one can find eight peaks in total.

(iii) For $q>1$ in the heating phase, one can check that the energy density away from the peaks approaches $-\left(q^{2} \pi c / 6 L^{2}\right)$ exponentially in time. It becomes "cooler" than the initial Casimir energy density $-\left(\pi c / 6 L^{2}\right)$. This result can be viewed as a dynamical Casimir effect [30-32].

\section{Entanglement pattern}

A more interesting question is how different energy peaks are entangled in the heating phase. Since we choose the boundary condition to be periodic and the initial state as the ground state of $H_{0}$, the state remains a tensor product of the chiral and antichiral components. It immediately follows that the entanglement entropy between energy 


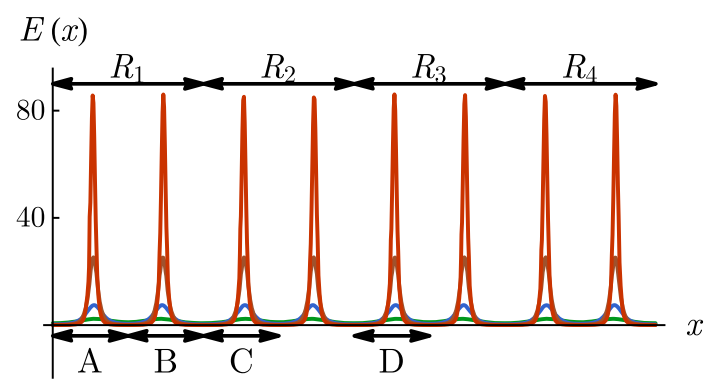

(a)

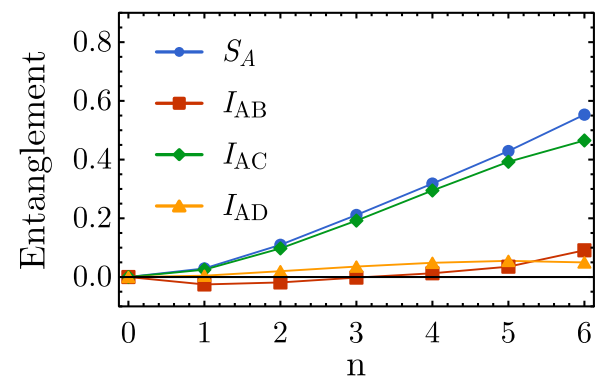

(b)

FIG. 14. (a) CFT calculation for the energy density. The system size is $L=2 \pi$, and the central charge $c=1$. We choose $q=4$, and $T_{0}=0.92 \mathrm{~L} / q$ and $T_{1}=0.1 \mathrm{~L} / q$ so that the system is in the heating phase. (b) Lattice calculation for the mutual information. We simulate complex free fermion on a 502-site chain, $q=4, T_{0}=0.92 \mathrm{~L} / q$, and $T_{1}=0.1 \mathrm{~L} / q$. The choice of subsystem $A, B, C$, or $D$ is consistent with (a). All the quantities are subtracted by their initial values. The data for $n>6$ are spoiled by the lattice effect.

peaks with different chirality does not grow. The entanglement among peaks with the same chirality requires more detailed analysis.

We first study the entanglement entropy of a single interval $\left[x_{1}, x_{2}\right]$, which is related to the correlation function of two twist operators $\left\langle\psi(t)\left|\mathcal{T}_{m}\left(x_{1}\right) \mathcal{T}_{m}\left(x_{2}\right)\right| \psi(t)\right\rangle$. Given the recipe above, it can be mapped to the following twopoint function on the complex plane:

$$
\frac{\left(\frac{\partial z_{1, n}}{\partial z_{1}}\right)^{h_{m}}\left(\frac{\partial \bar{z}_{1, n}}{\partial \bar{z}_{1}}\right)^{h_{m}}\left(\frac{\partial z_{2, n}}{\partial z_{2}}\right)^{h_{m}}\left(\frac{\partial \bar{z}_{2, n}}{\partial \bar{z}_{2}}\right)^{h_{m}}}{\left(z_{1, n}^{1 / q}-z_{2, n}^{1 / q}\right)^{2 h_{m}}\left(\bar{z}_{1, n}^{1 / q}-\bar{z}_{2, n}^{1 / q}\right)^{2 h_{m}}},
$$

where we keep only the time-dependent parts and $z_{j, n}$ and $\bar{z}_{j, n}$ denote the coordinates on the $q$-sheet Riemann surface after $n$-cycle driving. The result depends on whether there are chiral or antichiral energy peaks between the $x_{1}$ and $x_{2}$. If there are no energy peaks between $x_{1}$ and $x_{2}$, then $z_{1, n}$ and $z_{2, n}$ flow to the stable fixed point on the same sheet such that $z_{1, n}^{1 / q}-z_{2, n}^{1 / q}$ becomes exponentially small with time, which exactly cancels the time dependence from the $\partial z_{j, n} / \partial z_{j}$ prefactor. On the other hand, if there is a chiral energy peak between $x_{1}$ and $x_{2}, z_{1, n}$ and $z_{2, n}$ go to different Riemann sheets such that $z_{1, n}^{1 / q}-z_{2, n}^{1 / q}$ becomes an $\mathcal{O}(1)$ number at late time and the whole quantity has nontrivial time dependence. A similar argument works for $\bar{z}_{1, n}, \bar{z}_{2, n}$. Consequently, the entanglement entropy for a single region $\left[x_{1}, x_{2}\right]$ has a similar behavior as that shown in Eq. (33):

$$
S(t=n T)-S(0)= \begin{cases}\mathcal{O}(1) & {\left[x_{1}, x_{2}\right] \text { does not include peaks, }} \\ -\frac{c}{6} n \log \eta & {\left[x_{1}, x_{2}\right] \text { includes one peak, }} \\ -\frac{c}{3} n \log \eta & {\left[x_{1}, x_{2}\right] \text { includes both chiral and antichiral peaks. }}\end{cases}
$$

To determine the structure of the entanglement, such as whether it has bipartite entanglement or multipartite entanglement, we need to examine the mutual information between different peaks.

For example, let us consider the mutual information between $A$ and $C$, as depicted in Fig. 14(a), which covers two nearest-neighbor chiral peaks (ignoring the antichiral ones). The entanglement entropy $S_{A C}$ is related to the correlation function of four twist operators:

$$
\begin{gathered}
\frac{\prod_{j=1}^{4}\left(\frac{\partial z_{j, n}}{\partial z_{j}}\right)^{h_{m}}}{\left(z_{1, n}^{1 / q}-z_{4, n}^{1 / q}\right)^{2 h_{m}}\left(z_{2, n}^{1 / q}-z_{3, n}^{1 / q}\right)^{2 h_{m}}} F(\rho), \\
\rho=\frac{\left(z_{1, n}^{1 / q}-z_{4, n}^{1 / q}\right)\left(z_{2, n}^{1 / q}-z_{3, n}^{1 / q}\right)}{\left(z_{1, n}^{1 / q}-z_{2, n}^{1 / q}\right)\left(z_{4, n}^{1 / q}-z_{3, n}^{1 / q}\right)},
\end{gathered}
$$

where the antiholomorphic component is irrelevant to our discussion and thus ignored in the expression, $\rho$ is the cross ratio, and $F(\rho)$ is the conformal block. In the long-time limit, $z_{2, n}$ and $z_{3, n}$ flow to the same fixed point so that $z_{2, n}^{1 / q}-z_{3, n}^{1 / q}$ as well as $\rho$ becomes exponentially small while $z_{1, n}^{1 / q}-z_{4, n}^{1 / q}$ remains finite. This result implies that $S_{A C}$ linearly grows with time as $-(c / 6) n \log \eta$, so does the mutual information

$$
I_{A C}(t=n T)-I_{A C}(0)=-\frac{c}{6} n \log \eta .
$$

On the contrary, if we consider the mutual information between $A$ and $D$, as depicted in Fig. 14(a), the same analysis yields $S_{A D}(t=n T)-S_{A D}(0)=-(c / 3) n \log \eta$ such that the mutual information $I_{A D}$ does not grow at all. 


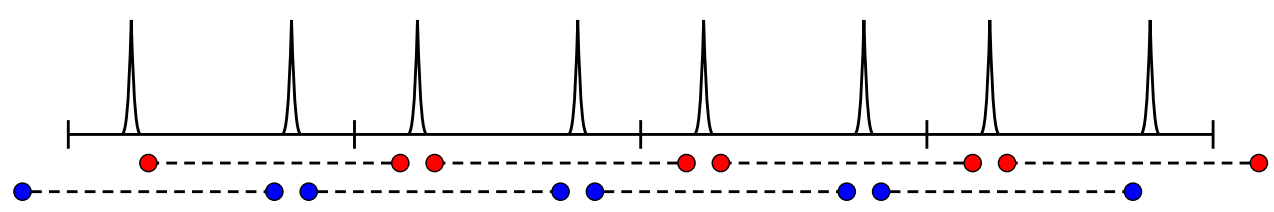

FIG. 15. A cartoon of the entanglement pattern for $q=4$ with a periodic boundary condition. Red and blue stand for two different chiralities. Each peak is entangled with its nearest neighbor with the same chirality or color.

Therefore, the system develops only bipartite entanglement; see Fig. 15 for an illustration of the pattern. Every two nearest-neighbor and only nearest-neighbor peaks of the same chirality share Bell pairs with each other. We point out that Fig. 15 is a stroboscopic picture; all the energy peaks as well as Bell pairs keep moving toward the left or right in each cycle. If we choose $(k-1) l<T_{0}<k l$, each energy peak can move from one subregion to the $k$ th subregion on its left or right and comes back to its original position only after every $q / \operatorname{gcd}(q, k)$ cycles of driving. This generalization to the peak-switching phenomena is first discussed in Sec. III C. We also simulate a free fermion on the lattice. The results are shown in Fig. 14(b), which supports our CFT argument. The deviation comes from the lattice effect.

\section{SUMMARY}

In this paper, we present a detailed study as well as a generalization of the Floquet CFT introduced in Ref. [34]. The phase diagram obtained in that paper can be understood by mapping the problem to a Floquet harmonic oscillator. The reason for such a mapping arises from the fact that these two problems share a $\mathfrak{s} \mathfrak{l}(2, \mathbb{R})$ algebra and the classification of dynamics becomes the classification of the linear combination of $\operatorname{SL}(2, \mathbb{R})$ generators. Because of that, although we use the entanglement entropy and total energy to explicitly determine the phase diagram, the calculation of which depends on the choice of the initial state, the result is actually a property of the driving Hamiltonian and does not depend on the initial state choice.

In the nonheating phase, the energy profile and total energy keep oscillating. In the heating phase, although the energy increases exponentially fast, the system is heated in an extremely nonuniform way; i.e., only two points absorb the heat. What is more, all the entanglement entropy is also shared by these two peaks. These peaks are determined by the fixed points of the relevant Möbius transformation that is defined by the dynamics. On the phase boundary between the heating and nonheating phases, we still observe two peaks, but the total energy increases only quadratically with time.

Although the question of whether the system absorbs energy relies on the detailed calculation, the energy density and entanglement structure can be understood by a quasiparticle picture. The questions of how energy distributes can be mapped to solving a pure classical motion. Inspired by this picture, we find a relation between the total energy and the entanglement between the two peaks, $E(t) \propto$ $c \exp [(6 / c) S(t)]$, which says the quasiparticle carries much more energy than entanglement. Such a relation, as contrary to the classic Cardy formula, is a clear manifestation of a nonequilibrium state. It will be interesting to understand whether this relation is special to this setup that involves only $\operatorname{SL}(2, \mathbb{R})$ or is true for more general cases.

To make some connection to the real experiment, we examine the robustness of all these features against random driving. Even if we add tiny randomness to the driving period, the nonheating phase completely disappears and we have only the heating phase, where the total energy grows exponentially with time. After we know whether the total energy grows, the energy density can be analyzed perturbatively. If we start from a $\left(T_{0}, T_{1}\right)$ that is deep inside the nonheating phase and turn on the randomness, the energy density peaks near the boundary. This peak is because the quasiparticles move incoherently and have smaller velocity near the boundary. On the other hand, if we start from a $\left(T_{0}, T_{1}\right)$ that is deep inside the heating phase and turn on moderate randomness, we expect the energy peaks to remain although they are smeared out a little.

Most of the phenomena above, in particular, including the existence of heating and nonheating phases and the features about the energy profile, not only hold for this special setup but should also occur for any generic Floquet driving that only uses Virasoro generators as the Hamiltonian. The reason is that this type of Floquet driving can always be thought of as a conformal mapping on the complex plane. The energy density calculation to a large extent can be reduced to the problem of finding fixed points of the conformal mapping. If none of the fixed points is on the unit circle, the system must be at the nonheating phase and energy density just oscillates. Once it has a repulsive fixed point on the unit circle, we see two energy peaks, one of which is purely chiral and the other is antichiral. The system is generically heated up. If there is also an attractive fixed point on the unit circle, the energy density decreases to make the energy peaks sharper and sharper. For this case, one has to do a more detailed calculation to determine whether the system is heating or not. Consequently, the problem of classifying dynamics is equivalent to the problem of classifying conformal mappings. This Floquet CFT using a sine-square deformed Hamiltonian is the first and simplest example that explicitly realizes this classification. It will be interesting to generalize this special 
setup to more general protocols and give a more thorough discussion on the connection between dynamics and geometry. Furthermore, since generic many-body Floquet drives do not have this geometric interpretation, it is also important to consider driving protocols that go beyond the conformal transformation paradigm, which can help develop a more general understanding of Floquet dynamics.

Another interesting problem is to consider the Floquet CFT from a thermal initial state at finite temperature $\beta^{-1}$, which is closely related with experiments. Since there are now three length scales, i.e., the total length $L$ of the system, the driving periods $T$, and the finite temperature $\beta$, then the time evolution of entanglement and energy density may exhibit more rich features, in particular, in the early time of driving. In the long-time limit, we expect there are still two phases, i.e., the heating and nonheating phases. One intuition is based on the quasiparticle picture as presented in Sec. III C. One can find that the existence of a fixed point or not in the solution of equation of motion, which determines the system is in heating or nonheating phases, is independent of the introduction of finite temperature $\beta^{-1}$. We expect these two phases to persist even if the system is prepared at a thermal initial state. We leave the detailed study in a future work.

The heating phase discussed here realizes a highly nonequilibrium state where entangled EPR pairs are continuously produced and localized at specific locations. Given the utility of entanglement as a resource for quantum information processing, experimental realization of the protocols discussed here may be desirable. Indeed, given the high tunability of ultracold atomic systems in optical lattices [67] and the ability to measure both energy and entanglement entropy [68], an important future direction is to find routes to implement these protocols in the lab.

\section{ACKNOWLEDGMENTS}

We thank Liujun Zou, Shang Liu, Andrew Potter, Xie Chen, Adam Nahum, Meng Cheng, Jie-Qiang Wu, Shinsei Ryu, Tsukasa Tada, and Ivar Martin for helpful discussions. Y. G. is supported by the Gordon and Betty Moore Foundation EPiQS Initiative through Grant No. GBMF4306 and DOE Grant No. DE-SC0019030. X. W. is supported by the Gordon and Betty Moore Foundations EPiQS initiative through Grant No. GBMF-4303 at MIT. A. V. and R. F. are supported by the DARPA DRINQS program (Grant No. D18AC00033) and by a Simons Investigator Award.

\section{APPENDIX A: PHASE DIAGRAM OF THE FLOQUET CFT}

The Floquet CFT defined in Sec. II is known to have two different phases, which is first shown in Ref. [34]. Here, we reproduce the phase diagram in Fig. 16 for the sake of being self-contained. The high-frequency regime is a nonheating

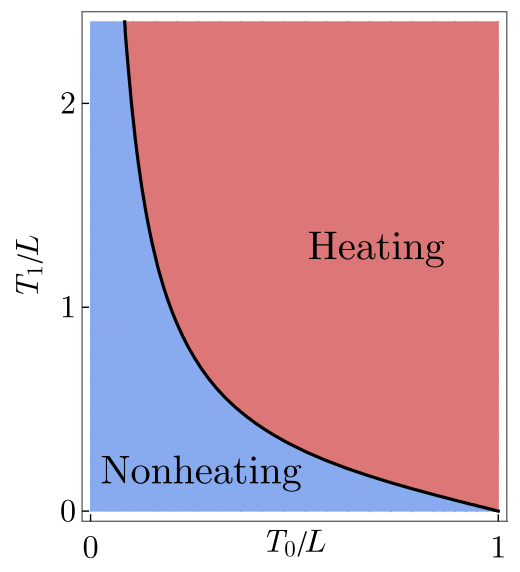

FIG. 16. Phase diagram of the Floquet CFT defined in Sec. II. The red regime is the heating phase, and the blue regime is the nonheating phase.

phase, where the entanglement entropy and energy oscillate. The low-frequency regime is a heating phase, where the entanglement entropy linearly grows and the energy exponentially grows. On the phase boundary, the entanglement entropy grows logarithmically and the energy grows quadratically. Figure 16 shows only one domain, and the phase diagram repeats itself when we increase $T_{0} / L$ with a period 1.

\section{APPENDIX B: ENTANGLEMENT GROWTH FOR A SUBSYSTEM AND BRANCH CUT CROSSING}

In this section, we present the details of the entanglement entropy growth calculation for a subsystem in the heating phase. As the early-time regime contains nonuniversal information, our analytical analysis focuses on the latetime regime (i.e., $n \gg 1$ ).

\section{Single entanglement cut}

For a given state on the interval $[0, L]$, we denote the (left) subsystem by $A=[0, x]$ and the corresponding reduced density matrix by $\rho_{A}$. Following Calabrese and Cardy's prescription [58], the $m$ th Rényi entropy

$$
S_{A}^{(m)}=\frac{1}{1-m} \log \operatorname{Tr} \rho_{A}^{m}
$$

can be computed using the twist operator $\mathcal{T}_{m}$, and the von Neumann entropy is the $m \rightarrow 1$ limit. More explicitly, the twist operator $\mathcal{T}_{m}$ is a primary with conformal dimension $h_{m}=\bar{h}_{m}=(c / 24)[m-(1 / m)]$, whose one-point function reproduces $\operatorname{Tr} \rho_{A}^{m}$ :

$$
\begin{aligned}
\operatorname{Tr} \rho_{A}^{m}= & \left(\frac{\partial z}{\partial w}\right)^{h_{m}}\left(\frac{\partial \bar{z}}{\partial \bar{w}}\right)^{h_{m}} \\
& \times\left(\frac{\partial z_{n}}{\partial z}\right)^{h_{m}}\left(\frac{\partial \bar{z}_{n}}{\partial \bar{z}}\right)^{h_{m}}\left\langle\mathcal{T}_{m}\left(z_{n}, \bar{z}_{n}\right)\right\rangle .
\end{aligned}
$$


The one-point correlation function $\left\langle\mathcal{T}_{m}\left(z_{n}, \bar{z}_{n}\right)\right\rangle$ in a boundary CFT can be mapped to a two-point function through the mirror trick on the whole plane, i.e.,

$$
\left\langle\mathcal{T}_{m}\left(z_{n}, \bar{z}_{n}\right)\right\rangle \propto\left(\frac{1}{4 \sqrt{z_{n}} \sqrt{\bar{z}_{n}}}\right)^{h_{m}}\left(\frac{1}{\sqrt{z_{n}}-\sqrt{\bar{z}_{n}}}\right)^{2 h_{m}} .
$$

Note that the derivative term in Eq. (B2) decreases exponentially as a function of $n$ in the long-time limit [69]:

$$
\begin{aligned}
& \left(\frac{\partial z_{n}}{\partial z}\right)^{h_{m}}\left(\frac{\partial \bar{z}_{n}}{\partial \bar{z}}\right)^{h_{m}} \\
& \quad \approx\left(\frac{\eta^{n}\left(\gamma_{1}-\gamma_{2}\right)^{2}}{\left(z-\gamma_{2}\right)\left(\bar{z}-\gamma_{2}\right)}\right)^{2 h_{m}} \quad \text { at } n \gg 1,
\end{aligned}
$$

which is related to the linear growth of entanglement entropy, while the behavior of $\left\langle\mathcal{T}_{m}\left(z_{n}, \bar{z}_{n}\right)\right\rangle$ depends on an interesting branch cut structure that leads to the spatial feature (the kink) of the entanglement entropy plotted in Fig. 7.

More explicitly, the branch cut arises from the factor $\left(\sqrt{z}_{n}-{\sqrt{z_{n}}}\right)$. The subtlety is that, although both $z_{n}$ and $\bar{z}_{n}$ flow to the same attractive fixed point $\gamma_{1}$ at the long-time limit, their square roots can be different due to the branch cut; i.e., the sign structure arises from the square root. To analyze the branch cut, let us use the two-layer Riemann sheet for $z$ and $\bar{z}$. At $t=0, z$ sits on the first sheet, while $\bar{z}$ sits on the second sheet [70]. Under the time evolution, we need to trace the trajectories of $z_{n}$ and $\bar{z}_{n}$; see Fig. 17 for an illustration of the trajectories for different scenarios. The upshot is that only when the entanglement cut is between the two energy peaks does the factor $\left(\sqrt{z}_{n}-{\sqrt{z_{n}}}\right)$ remain finite and, therefore, leads to the "bump" in the middle of Fig. 7.
To discuss this result in more detail, without loss of generality, we assume the chiral peak is on the left of the antichiral peak. Depending on the position of the entanglement cut, there are three different scenarios.

(1) $x<x_{C}$. - In this case, $z_{n}$ effectively crosses the branch cut. Therefore, when we take the square root of $z$ and $\bar{z}$, we have

$$
\begin{aligned}
& \sqrt{z_{n}}=-\gamma_{1}^{1 / 2}\left(1+\eta^{n} \frac{\gamma_{1}-\gamma_{2}}{2 \gamma_{1}} \frac{z-\gamma_{1}}{z-\gamma_{2}}\right), \\
& \sqrt{\bar{z}_{n}}=-\gamma_{1}^{1 / 2}\left(1+\eta^{n} \frac{\gamma_{1}-\gamma_{2}}{2 \gamma_{1}} \frac{\bar{z}-\gamma_{1}}{\bar{z}-\gamma_{2}}\right),
\end{aligned}
$$

so that their difference is exponentially small:

$$
\sqrt{z_{n}}-\sqrt{\bar{z}_{n}}=-\eta^{n} \frac{\gamma_{1}-\gamma_{2}}{2 \gamma_{1}^{1 / 2}} \frac{(z-\bar{z})\left(\gamma_{1}-\gamma_{2}\right)}{\left(z-\gamma_{2}\right)\left(\bar{z}-\gamma_{2}\right)} .
$$

This $\eta^{n}$ dependence exactly cancels the $\eta^{n}$ dependence in the derivative term. Therefore, the whole quantity and the entanglement entropy, to the leading order, does not grow with time.

(2) $x_{C}<x<x_{A}$. - In this case, $z_{n}$ and $\bar{z}_{n}$ do not cross the branch cut. When we calculate the square root, we have

$$
\begin{aligned}
& \sqrt{z_{n}}=\gamma_{1}^{1 / 2}\left(1+\eta^{n} \frac{\gamma_{1}-\gamma_{2}}{2 \gamma_{1}} \frac{z-\gamma_{1}}{z-\gamma_{2}}\right), \\
& \sqrt{\bar{z}_{n}}=-\gamma_{1}^{1 / 2}\left(1+\eta^{n} \frac{\gamma_{1}-\gamma_{2}}{2 \gamma_{1}} \frac{\bar{z}-\gamma_{1}}{\bar{z}-\gamma_{2}}\right),
\end{aligned}
$$

and their difference converges to $2 \gamma_{1}^{1 / 2}$ at the late time:

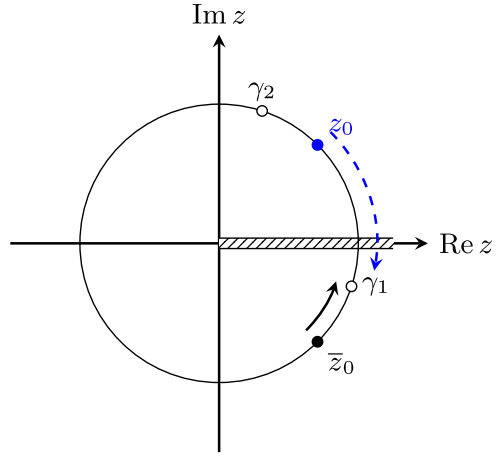

(a)

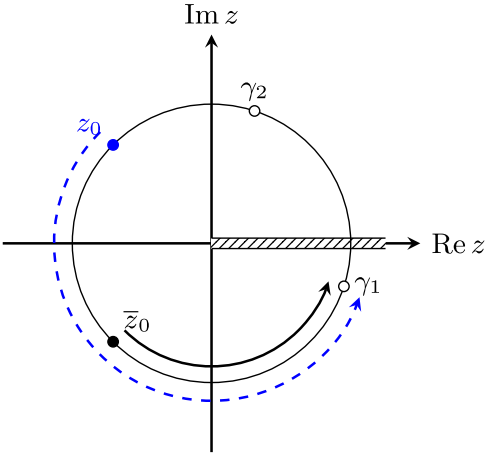

(b)

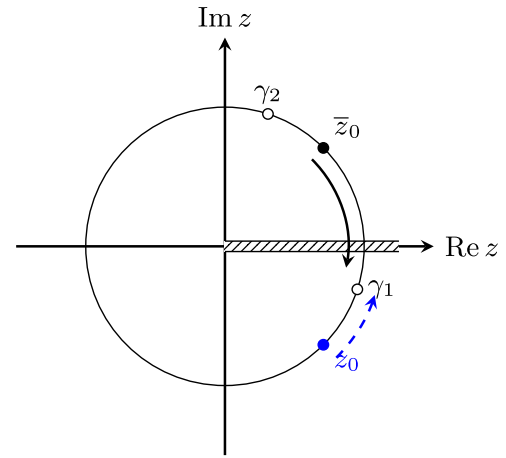

(c)

FIG. 17. Schematic plots for the stroboscopic trajectories of $z_{n}$ and $\bar{z}_{n}$ on a unit circle. Hollow dots are the fixed points, from which we know the chiral peak is on the left of the antichiral peak, i.e., $x_{C}<x_{A}$. Blue and black dots are the initial positions of $z_{n}$ and $\bar{z}_{n}$, respectively. In each cycle, both $z_{n}$ and $\bar{z}_{n}$ in each cycle wind around the unit circle counterclockwise and stop at the next position, with their stroboscopic positions represented by the blue dashed line and black line, respectively. The dashed bar denotes the branch cut. (a) $x<x_{C}$. The blue line crosses the branch cut, meaning that $z_{n}$ has a relative branch cut crossing to $\bar{z}_{n}$; therefore, they stay on the same Riemann sheet in the long-time limit. (b) $x_{C}<x<x_{A}$. Neither line crosses the branch cut; thus, $z_{n}$ and $\bar{z}_{n}$ remain on different sheets. (c) $x>x_{A}$. This time, it is the black line that crosses the branch cut; thus, $z_{n}$ and $\bar{z}_{n}$ still end up getting on the same Riemann sheet. 


$$
\sqrt{z_{n}}-\sqrt{\bar{z}_{n}}=2 \gamma_{1}^{1 / 2}+\mathcal{O}\left(\eta^{n}\right) .
$$

Therefore, the whole quantity depends on time through the $\eta^{n}$ in the derivative term. After taking the logarithm and $m \rightarrow 1$ limit, we can show that the entanglement entropy grows linearly with time:

$$
S_{A}(t)=-\frac{c}{6} n \log \eta,
$$

the slope of which is independent of $x$.

(3) $x>x_{A}$. - In this case, $\bar{z}_{n}$ crosses the branch cut and all the calculation becomes the same as the first case. Therefore, the entanglement does not grow with time, either.

These scenarios explain the kinks that we observe in Fig. 7.

\section{Entanglement entropy between two halves}

For the special case $x=L / 2$, we have $z=\bar{z}=e^{2 \pi x / L}=$ -1 and $z_{n}=\bar{z}_{n}$ for any integer $n$. As a result, $z_{n}$ and $\bar{z}_{n}$ are always on the opposite Riemann surfaces, i.e., $\sqrt{z}_{n}=-\sqrt{\bar{z}_{n}}$. Hence, the expression for $\operatorname{Tr} \rho_{A}^{m}$ can be simplified as

$$
\begin{aligned}
\operatorname{Tr} \rho_{A}^{m} & \propto\left(\frac{\pi}{L}\right)^{2 h_{m}}\left(\frac{\partial z_{n}}{\partial z}\right)^{2 h_{m}}\left(\frac{-1}{z_{n}}\right)^{h_{m}}\left(\frac{1}{z_{n}}\right)^{h_{m}} \\
& \propto\left(\frac{\pi}{L} \frac{\partial z_{n}}{\partial z} \frac{1}{z_{n}}\right)^{2 h_{m}},
\end{aligned}
$$

where all the nonuniversal constants are dropped. Recalling our expression Eq. (11) for $z_{n}$ and plugging in the initial condition that $z=-1$, we can write the universal part of the entanglement entropy as

$$
\begin{aligned}
S_{A}(t)= & \frac{c}{6} \log \left[\frac{L}{\pi} \frac{(A-B)(C-D)}{A D-B C}\right] \\
& +(\text { nonuniversal term }),
\end{aligned}
$$

where the nonuniversal term refers to the $n$-independent contributions. In the nonheating phase, the universal part oscillates in a similar fashion as the total energy. In the heating phase, the leading growing behavior of the entanglement entropy is given as follows:

$S_{A, \text { heating }}(t) \sim \frac{c}{6} \log \left[\frac{L}{\pi} \frac{\left(1+\gamma_{2}\right)^{2} \gamma_{1}}{\left(\gamma_{1}-\gamma_{2}\right)^{2}} \eta^{-n}\right]$ at $n \gg 1$.

For the critical phase, we have the following logarithmic growing:

$$
S_{A, \text { critical }}(t) \sim \frac{c}{6} \log \left[\frac{L}{\pi}(1+\gamma)^{2} \beta^{2} \gamma n^{2}\right] \quad \text { at } n \gg 1 .
$$

\section{Two entanglement cuts}

In this section, we present the details of computing the entanglement entropy for a subsystem that does not end at the boundary, i.e., with ending points $x_{1}, x_{2} \in(0, L)$. In other words, we need to insert two twist operators:

$$
\begin{aligned}
\operatorname{Tr} \rho_{A}^{m}(t) & =C_{m}\left(x_{1}, x_{2}, t\right) \\
& =\left\langle\psi(t)\left|\mathcal{T}_{m}\left(x_{1}\right) \mathcal{T}_{m}\left(x_{2}\right)\right| \psi(t)\right\rangle \\
& =\left\langle G\left|\mathcal{T}_{m}\left(x_{1}, t\right) \mathcal{T}_{m}\left(x_{2}, t\right)\right| G\right\rangle .
\end{aligned}
$$

We follow the strategy in Sec. II to do the calculation first in the imaginary time and analytically continue to real time in the end. The $n$-dependent part of the above formula is given as follows:

$$
\begin{aligned}
& \prod_{j=1,2}\left(\frac{\partial z_{j, n}}{\partial z_{j}}\right)^{h_{m}}\left(\frac{\partial \bar{z}_{j, n}}{\partial \bar{z}_{j}}\right)^{h_{m}} \\
& \quad \times\left\langle\mathcal{T}_{m}\left(z_{1, n}, \bar{z}_{1, n}\right) \mathcal{T}_{m}\left(z_{2, n}, \bar{z}_{2, n}\right)\right\rangle .
\end{aligned}
$$

The mirror trick maps the two-point function $\left\langle\mathcal{T}_{m}\left(z_{1, n}\right.\right.$, $\left.\left.\bar{z}_{1, n}\right) \mathcal{T}_{m}\left(z_{2, n}, \bar{z}_{2, n}\right)\right\rangle$ in a boundary CFT to a four-point function on the whole plane without a boundary. The important $n$-dependent part is given by the following formula:

$$
\begin{aligned}
& \left\langle\mathcal{T}_{m}\left(z_{1, n}, \bar{z}_{1, n}\right) \mathcal{T}_{m}\left(z_{2, n}, \bar{z}_{2, n}\right)\right\rangle \\
& \quad \propto \frac{1}{\left(\sqrt{z_{1, n}}-\sqrt{z_{2, n}}\right)^{2 h_{m}}\left(\sqrt{\bar{z}_{1, n}}-\sqrt{\bar{z}_{2, n}}\right)^{2 h_{m}}} F(\rho),
\end{aligned}
$$

where $\rho$ is the cross ratio of the four $\sqrt{z_{n}}$, s defined as follows:

$$
\rho=\frac{\left(\sqrt{z_{1, n}}-\sqrt{z_{2, n}}\right)\left(\sqrt{\overline{z_{1, n}}}-\sqrt{\bar{z}_{2, n}}\right)}{\left(\sqrt{z_{1, n}}-\sqrt{\overline{z_{1, n}}}\right)\left(\sqrt{z_{2, n}}-\sqrt{\overline{z_{2, n}}}\right)} .
$$

$F(\rho)$ is a linear combination of the chiral conformal blocks with coefficients determined by the boundary condition.

While analytically continuing to the real time, the derivative term shows an exponential decrease as a function of $n$ (similar to the single entanglement cut case):

$$
\begin{aligned}
& \prod_{j=1,2}\left(\frac{\partial z_{j n}}{\partial z_{j}}\right)^{h_{m}}\left(\frac{\partial \bar{z}_{j n}}{\partial \bar{z}_{j}}\right)^{h_{m}} \\
& \quad \approx\left(\frac{\eta^{2 n}\left(\gamma_{1}-\gamma_{2}\right)^{4}}{\left(z_{1}-\gamma_{2}\right)\left(\bar{z}_{1}-\gamma_{2}\right)\left(z_{2}-\gamma_{2}\right)\left(\bar{z}_{2}-\gamma_{2}\right)}\right)^{2 h_{m}} .
\end{aligned}
$$

Note that the exponential decrease of the correlation function is related to the linearly growth of entanglement entropy.

For the analysis of the behavior of $\left\langle\mathcal{T}_{m}\left(z_{1, n}, \bar{z}_{1, n}\right) \times\right.$ $\left.\mathcal{T}_{m}\left(z_{2, n}, \bar{z}_{2, n}\right)\right\rangle$, there are two complications: First is the 
branch cut issue due to the $\sqrt{z}$ factor as we discuss before; the second is the potential divergence caused by $F(\rho)$. In the following, we show that $\rho$ flows to a final value $\rho_{\text {final }} \neq 1$ which, for different choices of $x_{1}$ and $x_{2}$, is either 0 or a constant finite value so that $F(\rho)$ converges to a constant nonzero value at late time and can be neglected.

Without loss of generality, let us assume the chiral peak is on the left of the antichiral peak. We fix $x_{2}$ to be between the two peaks so that $z_{2, n}$ and $\bar{z}_{2, n}$ stay on different Riemann sheets.

(1) $x_{1}<x_{C}$, the subsystem $A$ includes the chiral peak.-As discussed in Fig. 17, only $z_{1, n}$ crosses the branch cut during the time evolution. Therefore, at the late time, the leading terms of four $\sqrt{z}$ read

$$
\begin{aligned}
& \sqrt{z_{1, n}}=-\gamma_{1}^{1 / 2}\left(1+\eta^{n} \frac{\gamma_{1}-\gamma_{2}}{2 \gamma_{1}} \frac{z_{1}-\gamma_{1}}{z_{1}-\gamma_{2}}\right), \\
& \sqrt{\bar{z}_{1, n}}=-\gamma_{1}^{1 / 2}\left(1+\eta^{n} \frac{\gamma_{1}-\gamma_{2}}{2 \gamma_{1}} \frac{\bar{z}_{1}-\gamma_{1}}{\bar{z}_{1}-\gamma_{2}}\right), \\
& \sqrt{z_{2, n}}=\gamma_{1}^{1 / 2}\left(1+\eta^{n} \frac{\gamma_{1}-\gamma_{2}}{2 \gamma_{1}} \frac{z_{2}-\gamma_{1}}{z_{2}-\gamma_{2}}\right), \\
& \sqrt{\bar{z}_{2, n}}=-\gamma_{1}^{1 / 2}\left(1+\eta^{n} \frac{\gamma_{1}-\gamma_{2}}{2 \gamma_{1}} \frac{\bar{z}_{2}-\gamma_{1}}{\bar{z}_{2}-\gamma_{2}}\right) .
\end{aligned}
$$

These show that $\sqrt{z_{1, n}}-\sqrt{z_{2, n}}$ and $\sqrt{z_{2, n}}-\sqrt{\bar{z}_{2, n}}$ converge to $\pm 2 \sqrt{\gamma_{1}}$, while $\sqrt{\bar{z}_{1, n}}-\sqrt{\bar{z}_{2, n}}$ and $\sqrt{z_{1, n}}-\sqrt{\bar{z}_{1, n}}$ become exponentially small. The cross ratio $\rho$ converges to a $\mathcal{O}(1)$ value $\rho_{\text {final }}$ :

$$
\rho_{\text {final }}=\frac{\left(\bar{z}_{1}-\bar{z}_{2}\right)\left(z_{1}-\gamma_{2}\right)}{\left(z_{1}-\bar{z}_{1}\right)\left(\bar{z}_{2}-\gamma_{2}\right)} .
$$

The condition that neither $x_{1}$ or $x_{2}$ is at the energy peaks implies $\rho_{\text {final }} \neq 1$. Therefore, the conformal block term $F(\rho)$ converges only to an $\mathcal{O}(1)$ value and does not contribute to the $n$ dependence. As a result, the late-time behavior is controlled by the derivative terms, which leads to the linear growth behavior of the entanglement entropy:

$$
\begin{aligned}
S_{A}\left(x_{1}, x_{2}, t\right) & =\lim _{m \rightarrow 1} \frac{1}{1-m} \log C_{m}\left(x_{1}, x_{2}, t\right) \\
& \sim-\frac{c}{6} n \log \eta .
\end{aligned}
$$

The slope depends only on the central charge and the characteristic constant $\eta$ but not on the positions of entanglement cuts, as long as $x_{1}<x_{C}$.

(2) $x_{C}<x_{1}, x_{2}<x_{A}$, the subsystem is between the chiral and antichiral peaks.-None of the four $z$ coordinates have any relative branch cut crossing, and they can be assumed to remain on their original Riemann sheets during the whole time evolution. Hence, the late-time values of their square roots are

$$
\begin{aligned}
& \sqrt{z_{1, n}}=\gamma_{1}^{1 / 2}\left(1+\eta^{n} \frac{\gamma_{1}-\gamma_{2}}{2 \gamma_{1}} \frac{z_{1}-\gamma_{1}}{z_{1}-\gamma_{2}}\right), \\
& \sqrt{\bar{z}_{1, n}}=-\gamma_{1}^{1 / 2}\left(1+\eta^{n} \frac{\gamma_{1}-\gamma_{2}}{2 \gamma_{1}} \frac{\bar{z}_{1}-\gamma_{1}}{\bar{z}_{1}-\gamma_{2}}\right), \\
& \sqrt{z_{2, n}}=\gamma_{1}^{1 / 2}\left(1+\eta^{M} \frac{\gamma_{1}-\gamma_{2}}{2 \gamma_{1}} \frac{z_{2}-\gamma_{1}}{z_{2}-\gamma_{2}}\right), \\
& \sqrt{\bar{z}_{2, n}}=-\gamma_{1}^{1 / 2}\left(1+\eta^{M} \frac{\gamma_{1}-\gamma_{2}}{2 \gamma_{1}} \frac{\bar{z}_{2}-\gamma_{1}}{\bar{z}_{2}-\gamma_{2}}\right) .
\end{aligned}
$$

As a result, $\sqrt{z_{1, n}}-\sqrt{z_{2, n}}$ and $\sqrt{\bar{z}_{1, n}}-\sqrt{\bar{z}_{2, n}}$ become exponentially small so that the prefactor in Eq. (B16) cancels the time dependence in the derivative term. $\sqrt{z_{1, n}}-\sqrt{\bar{z}_{1, n}}$ and $\sqrt{z_{2, n}}-\sqrt{z_{2, n}}$ converge to $2 \sqrt{\gamma_{1}}$ so that the cross ratio $\rho$ now converges to $\rho=0$. However, in the way that we write Eq. (B16), the conformal block term is already regularized at $\rho=0$ and takes an $\mathcal{O}(1)$ value depending on the fusion from two twist operators to the identity channel. In this limit, the boundary two-point function should be reduced to a bulk twopoint function, which is nonzero in our case. This result implies $F(\rho=0)$ is a nonzero number and, thus, does not carry important time dependence. As a result, the late-time behavior of the entanglement entropy, to the leading order, is independent of time.

(3) $x_{1}>x_{A}$, the subsystem includes the antichiral peak.-Now, the $\bar{z}_{1}$ crosses the branch cut and the entanglement entropy linear grows again, which is the same as the first case.

The analysis above confirms that the entanglement indeed comes only from the two energy peaks, which verifies our quasiparticle picture from a technical side.

\section{APPENDIX C: RANDOM DRIVING MATHIEU OSCILLATOR}

In this section, we discuss the random driving Mathieu oscillator. The classical Newton's equation for a Mathieu oscillator is

$$
\ddot{x}(t)+\left(1+h \cos \frac{2 \pi t}{T}\right) x(t)=0 .
$$

$h$ controls the amplitude of the driving force, and $T$ is the driving period. The intrinsic period of the harmonic oscillator is $2 \pi$. For a weak driving force $h \ll 1$, the first smallest unstable driving period is $T_{\text {unstable }}=\pi$. The system is stable (nonheating) for any $T<T_{\text {unstable. These are }}$ shown in Fig. 18(a).

For a random driving Mathieu oscillator, we let the driving period $T$ uniformly distribute in an interval

$$
T=\bar{T}+\delta T, \quad \delta T=[-\alpha, \alpha],
$$

where $\alpha$ controls the strength of the randomness. In each cycle, we randomly choose a $T$ from the distribution and evolve the system accordingly. The final results are 


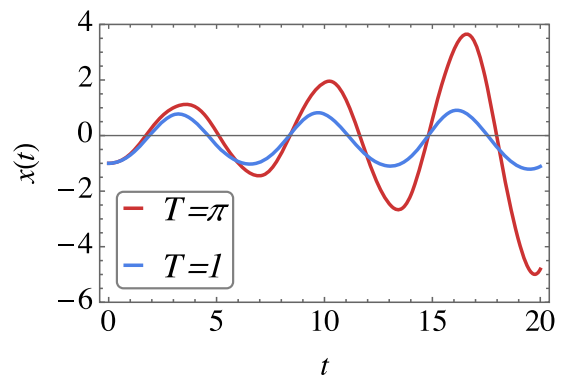

(a)

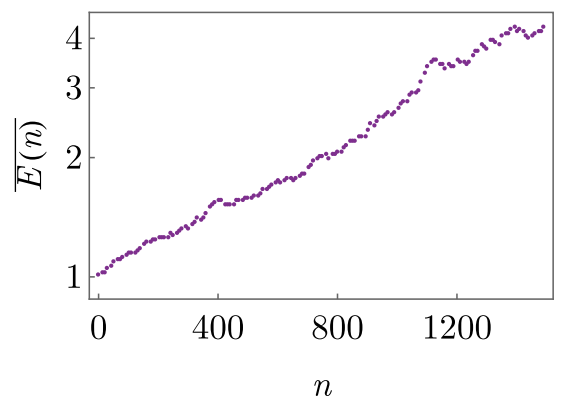

(b)

FIG. 18. Dynamics of a Mathieu oscillator without and with random driving. (a) Mathieu oscillator under a weak driving force is stable for $T<1$ and unstable for $T=\pi$. We choose $h=0.2$. (b) For random driving, the energy grows exponentially even for small randomness. We choose $h=0.2, \bar{T}=1$, and $\alpha=0.1$, Each data point is averaged over 100 times.

averaged over "disorder." If we choose $\bar{T}<T_{\text {unstable }}$ and $\alpha \ll 1$, we find that the averaged energy grows exponentially with time, as is demonstrated in Fig. 18(b).

\section{APPENDIX D: SPATIAL STRUCTURES IN LATTICE CALCULATION}

In this section, we discuss the time evolution of the energy density profile and the entanglement entropy density in the heating phase observed in a lattice calculation. The protocol is the one introduced by Ref. [34] also

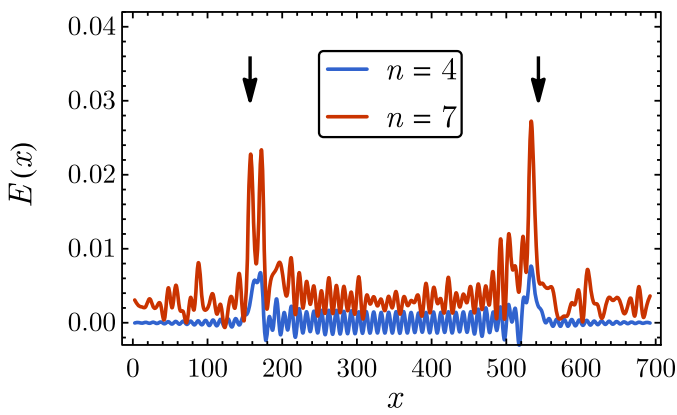

(a)

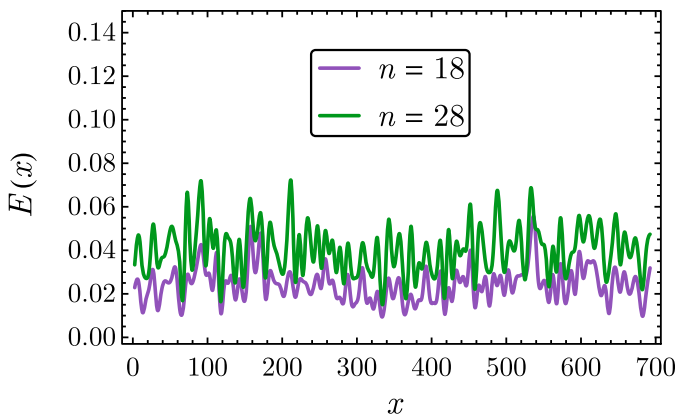

(c) reviewed in Sec. II. A simulation with the generalized setup in Sec. V yields the same results and, thus, is not included.

We simulate a complex free fermion on an open chain with only nearest-neighbor hopping at half filling. The results are shown in Fig. 19, with Figs. 19(a) and 19(b) being the early-time regime and Figs. 19(c) and 19(d) being the late-time regime. In the early-time regime, both quantities show growing sharp peaks, whose positions are consistent with the CFT prediction as indicated by the arrows in Figs. 19(a) and 19(b). However, in the late

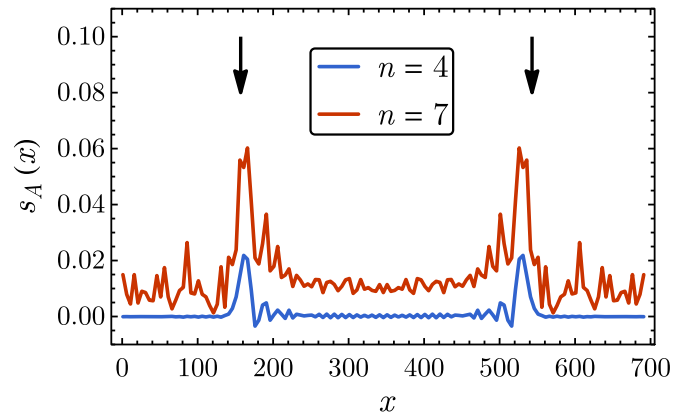

(b)

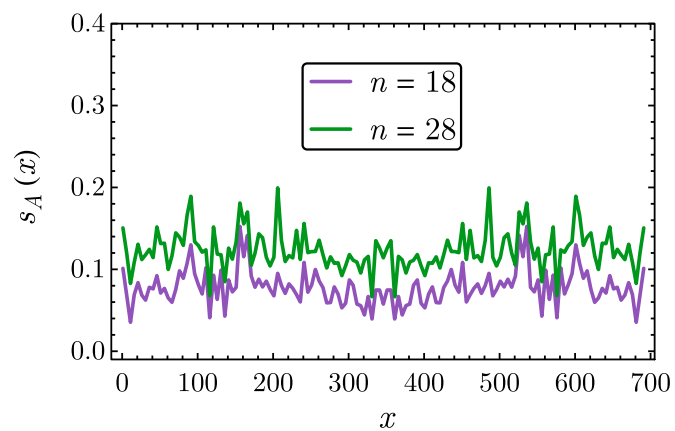

(d)

FIG. 19. Spatial structure in the lattice simulation. We choose the system size $L=700$ and driving parameters $T_{0} / L=0.9$ and $T_{1} / L=0.2$. We choose $\delta=5$ when performing the average. $n$ denotes the number of driving cycles. The black arrows in (a) and (b) indicate the peak positions predicted by the CFT calculation. (a) Energy density at early time, (b) Entropy density at early time,

(c) Energy density at late time, and (d) Entropy density at late time. 
time, as more and more excitations are created, the dynamics of the lattice system cannot be approximated by a CFT. One sees the spatial structure showing strong oscillation with time. The peaks also stop growing and finally give way to a smeared profile, as depicted in Figs. 19(c) and 19(d). Determining the timescale at which the prediction of conformal field theory begins to diverge from lattice calculations is a subtle question. Here, we simply note that, on comparing the energy or entropy density in this model using the parameters as in Fig. 19, the breakdown occurs around $n \sim 10$. It is roughly the timescale for $E_{\text {total }} / t \sim \mathcal{O}(1)$ with $t$ being the hopping strength. On the other hand, the half-system entropy can agree with the CFT calculation for longer times, which in this model breaks down at $n \sim 30$ (using the same parameters as Fig. 19).

We close this section with some technical details of how the data are extracted from the numerics. The energy density $E(x)$ is obtained by computing the expectation value of the hopping term $-t\left\langle c_{i}^{\dagger} c_{i+1}+\right.$ H.c. $\rangle$. We also perform an average over the nearest few sites to obtain a relatively smooth curve, i.e., $E(x)=\sum_{k=-\delta}^{\delta}-$ $t\left\langle c_{x+k}^{\dagger} c_{x+k+1}+\right.$ H.c. $\rangle$. The slight asymmetry of the plots with respect to the middle of the system is due to this average. Choosing different $\delta$ leads to results with the same qualitative features. The entanglement entropy density $s_{A}(x)$ is obtained by computing the entanglement entropy for the subsystem $[x-\delta, x+\delta]$. A similar average is also performed to obtain a smooth curve.

[1] L. Jiang, T. Kitagawa, J. Alicea, A. R. Akhmerov, D. Pekker, G. Refael, J. I. Cirac, E. Demler, M. D. Lukin, and P. Zoller, Majorana Fermions in Equilibrium and Driven Cold Atom Quantum Wires, Phys. Rev. Lett. 106, 220402 (2011).

[2] T. Kitagawa, E. Berg, M. Rudner, and E. Demler, Topological Characterization of Periodically Driven Quantum Systems, Phys. Rev. B 82, 235114 (2010).

[3] M. S. Rudner, N. H. Lindner, E. Berg, and M. Levin, Anomalous Edge States and the Bulk-Edge Correspondence for Periodically Driven Two-Dimensional Systems, Phys. Rev. X 3, 031005 (2013).

[4] C. W. von Keyserlingk, V. Khemani, and S. L. Sondhi, Absolute Stability and Spatiotemporal Long-Range Order in Floquet Systems, Phys. Rev. B 94, 085112 (2016).

[5] D. V. Else and C. Nayak, Classification of Topological Phases in Periodically Driven Interacting Systems, Phys. Rev. B 93, 201103(R) (2016).

[6] A. C. Potter, T. Morimoto, and A. Vishwanath, Classification of Interacting Topological Floquet Phases in One Dimension, Phys. Rev. X 6, 041001 (2016).

[7] R. Roy and F. Harper, Abelian Floquet Symmetry-Protected Topological Phases in One Dimension, Phys. Rev. B 94, 125105 (2016).
[8] H. C. Po, L. Fidkowski, T. Morimoto, A. C. Potter, and A. Vishwanath, Chiral Floquet Phases of Many-Body Localized Bosons, Phys. Rev. X 6, 041070 (2016).

[9] R. Roy and F. Harper, Periodic Table for Floquet Topological Insulators, Phys. Rev. B 96, 155118 (2017).

[10] F. Harper and R. Roy, Floquet Topological Order in Interacting Systems of Bosons and Fermions, Phys. Rev. Lett. 118, 115301 (2017).

[11] H. C. Po, L. Fidkowski, A. Vishwanath, and A. C. Potter, Radical Chiral Floquet Phases in a Periodically Driven Kitaev Model and Beyond, Phys. Rev. B 96, 245116 (2017).

[12] I.-D. Potirniche, A. C. Potter, M. Schleier-Smith, A. Vishwanath, and N. Y. Yao, Floquet Symmetry-Protected Topological Phases in Cold-Atom Systems, Phys. Rev. Lett. 119, 123601 (2017).

[13] T. Morimoto, H.C. Po, and A. Vishwanath, Floquet Topological Phases Protected by Time Glide Symmetry, Phys. Rev. B 95, 195155 (2017).

[14] L. Fidkowski, H. C. Po, A. C. Potter, and A. Vishwanath, Interacting Invariants for Floquet Phases of Fermions in Two Dimensions, Phys. Rev. B 99, 085115 (2019).

[15] V. Khemani, A. Lazarides, R. Moessner, and S. L. Sondhi, Phase Structure of Driven Quantum Systems, Phys. Rev. Lett. 116, 250401 (2016).

[16] D. V. Else, B. Bauer, and C. Nayak, Floquet Time Crystals, Phys. Rev. Lett. 117, 090402 (2016).

[17] C. W. von Keyserlingk and S. L. Sondhi, Phase Structure of One-Dimensional Interacting Floquet Systems. I. Abelian Symmetry-Protected Topological Phases, Phys. Rev. B 93, 245145 (2016).

[18] C. W. von Keyserlingk and S. L. Sondhi, Phase Structure of One-Dimensional Interacting Floquet Systems. II. SymmetryBroken Phases, Phys. Rev. B 93, 245146 (2016).

[19] D. V. Else, B. Bauer, and C. Nayak, Prethermal Phases of Matter Protected by Time-Translation Symmetry, Phys. Rev. X 7, 011026 (2017)

[20] N. Y. Yao, A. C. Potter, I.-D. Potirniche, and A. Vishwanath, Discrete Time Crystals: Rigidity, Criticality, and Realizations, Phys. Rev. Lett. 118, 030401 (2017).

[21] S. Choi, J. Choi, R. Landig, G. Kucsko, H. Zhou, J. Isoya, F. Jelezko, S. Onoda, H. Sumiya, V. Khemani et al., Observation of Discrete Time-Crystalline Order in a Disordered Dipolar Many-Body System, Nature (London) 543, 221 (2017).

[22] J. Zhang, P. W. Hess, A. Kyprianidis, P. Becker, A. Lee, J. Smith, G. Pagano, I. D. Potirniche, A. C. Potter, A. Vishwanath, N. Y. Yao, and C. Monroe, Observation of a Discrete Time Crystal, Nature (London) 543, 217 (2017).

[23] N. Y. Yao, C. Nayak, L. Balents, and M. P. Zaletel, Classical Discrete Time Crystals, Nat. Phys. 16, 438 (2020).

[24] L. D'Alessio and M. Rigol, Long-Time Behavior of Isolated Periodically Driven Interacting Lattice Systems, Phys. Rev. X 4, 041048 (2014).

[25] P. Ponte, Z. Papić, F. Huveneers, and D. A. Abanin, ManyBody Localization in Periodically Driven Systems, Phys. Rev. Lett. 114, 140401 (2015).

[26] D. A. Abanin, W. De Roeck, and F. Huveneers, Theory of Many-Body Localization in Periodically Driven Systems, Ann. Phys. (Amsterdam) 372, 1 (2016). 
[27] D. A. Abanin, W. De Roeck, and F. Huveneers, Exponentially Slow Heating in Periodically Driven Many-Body Systems, Phys. Rev. Lett. 115, 256803 (2015).

[28] D. Abanin, W. De Roeck, W. W. Ho, and F. Huveneers, A Rigorous Theory of Many-Body Prethermalization for Periodically Driven and Closed Quantum Systems, Commun. Math. Phys. 354, 809 (2017).

[29] D. A. Abanin, W. De Roeck, W. W. Ho, and F. Huveneers, Effective Hamiltonians, Prethermalization, and Slow Energy Absorption in Periodically Driven Many-Body Systems, Phys. Rev. B 95, 014112 (2017).

[30] C. K. Law, Resonance Response of the Quantum Vacuum to an Oscillating Boundary, Phys. Rev. Lett. 73, 1931 (1994).

[31] V. V. Dodonov and A. B. Klimov, Generation and Detection of Photons in a Cavity with a Resonantly Oscillating Boundary, Phys. Rev. A 53, 2664 (1996).

[32] I. Martin, Floquet Dynamics of Classical and Quantum Cavity Fields, Ann. Phys. (Amsterdam) 405, 101 (2019).

[33] W. Berdanier, M. Kolodrubetz, R. Vasseur, and J. E. Moore, Floquet Dynamics of Boundary-Driven Systems at Criticality, Phys. Rev. Lett. 118, 260602 (2017).

[34] X. Wen and J.-Q. Wu, Floquet Conformal Field Theory, arXiv:1805.00031.

[35] A. A. Belavin, A. M. Polyakov, and A. B. Zamolodchikov, Infinite Conformal Symmetry in Two-Dimensional Quantum Field Theory, Nucl. Phys. B241, 333 (1984).

[36] P. Francesco, P. Mathieu, and D. Sénéchal, Conformal Field Theory (Springer, New York, 2012).

[37] T. Hikihara and T. Nishino, Connecting Distant Ends of One-Dimensional Critical Systems by a Sine-Square Deformation, Phys. Rev. B 83, 060414(R) (2011).

[38] I. Maruyama, H. Katsura, and T. Hikihara, Sine-Square Deformation of Free Fermion Systems in One and Higher Dimensions, Phys. Rev. B 84, 165132 (2011).

[39] H. Katsura, Sine-Square Deformation of Solvable Spin Chains and Conformal Field Theories, J. Phys. A 45, 115003 (2012).

[40] N. Ishibashi and T. Tada, Infinite Circumference Limit of Conformal Field Theory, J. Phys. A 48, 315402 (2015).

[41] N. Ishibashi and T. Tada, Dipolar Quantization and the Infinite Circumference Limit of Two-Dimensional Conformal Field Theories, Int. J. Mod. Phys. A 31, 1650170 (2016).

[42] K. Okunishi, Sine-Square Deformation and Möbius Quantization of 2D Conformal Field Theory, Prog. Theor. Exp. Phys. 2016, 063A02 (2016).

[43] X. Wen, S. Ryu, and A. W. W. Ludwig, Evolution Operators in Conformal Field Theories and Conformal Mappings: Entanglement Hamiltonian, the Sine-Square Deformation, and Others, Phys. Rev. B 93, 235119 (2016).

[44] S. Tamura and H. Katsura, Zero-Energy States in Conformal Field Theory with Sine-Square Deformation, Prog. Theor. Exp. Phys. 2017, 113A01 (2017).

[45] T. Tada, Conformal Quantum Mechanics and Sine-Square Deformation, Prog. Theor. Exp. Phys. 2018, 061B01 (2018).

[46] X. Wen and J.-Q. Wu, Quantum Dynamics in Sine-Square Deformed Conformal Field Theory: Quench from Uniform to Nonuniform Conformal Field Theory, Phys. Rev. B 97, 184309 (2018).
[47] T. Tada, Time Development of Conformal Field Theories Associated with $l\{1\}$ and $l\{-1\}$ Operators, arXiv:1904 .12414

[48] I. MacCormack, A. Liu, M. Nozaki, and S. Ryu, Holographic Duals of Inhomogeneous Systems: The Rainbow Chain and the Sine-Square Deformation Model, J. Phys. A 52, 505401 (2019).

[49] Since the $H_{1}$ acts on the $z$ coordinate in a complicated way, we can instead look for a new coordinate $\chi$, on which $H_{1}$ acts as a simple dilation. Namely, we assume a coordinate change $\chi(z)$ and, accordingly, $T(z)=\chi^{\prime 2} T(\chi), H_{1}=$ $(2 \pi / L) \oint(d \chi / 2 \pi i)\left[-(1-z)^{2} / 2\right] \chi^{\prime} T(\chi)$. Requiring $H_{1}$ to generate a dilation amounts to the following condition: $-\left[(1-z)^{2} / 2\right] \chi^{\prime}=\chi \Rightarrow \frac{1}{2} \log \chi=[1 /(z-1)]+$ const. Under the evolution of $H_{1}, \chi$ goes to $\chi e^{2 \pi \tau_{1} / L}$, and, correspondingly, the $\tilde{z}$ transforms as $\left[1 /\left(z_{1}-1\right)\right]=[1 /(\tilde{z}-1)]+$ $\left(\pi \tau_{1} / L\right)$. Inserting $\tilde{z}=e^{2 \pi \tau_{0} / L} z$, we get Eq. (10).

[50] A. M. Perelomov and V.S. Popov, Group-Theoretical Aspects of the Variable Frequency Oscillator Problem, Theor. Math. Phys. 1, 275 (1969).

[51] V. Gritsev and A. Polkovnikov, Integrable Floquet Dynamics, SciPost Phys. 2, 021 (2017).

[52] S. Deng, Z.-Y. Shi, P. Diao, Q. Yu, H. Zhai, R. Qi, and H. $\mathrm{Wu}$, Observation of the Efimovian Expansion in ScaleInvariant Fermi Gases, Science 353, 371 (2016).

[53] Z.-Y. Shi, R. Qi, H. Zhai, and Z. Yu, Dynamic Super Efimov Effect, Phys. Rev. A 96, 050702(R) (2017).

[54] Another familiar example is the Bogoliubov transformation for bosons.

[55] Since $F_{(x, p)}$ is singular, its Jordan normal form has a nonzero off-diagonal element $F_{(x, p)}=P\left(\begin{array}{ll}1 & 1 \\ 0 & 1\end{array}\right) P^{-1}$. The offdiagonal element increases linearly with the driving cycles, i.e., $F_{(x, p)}^{n}=P\left(\begin{array}{ll}1 & n \\ 0 & 1\end{array}\right) P^{-1}$.

[56] Indeed, in Sec. II B, we reduce the operator evolution with an open boundary condition to the one with a periodic boundary condition using a contour deformation trick. The reason we start with an open boundary condition is that the ground state of $H_{0}$ is also an eigenstate of $H_{1}$ for the periodic boundary condition but not for the open boundary condition.

[57] Given $f(x)$, we can use its Fourier decomposition to rewrite it in terms of $z=e^{2 \pi i x / L}$ as $f(x)=\tilde{f}(z)$. Then, one can use the same technique as Reference [49] to show that $H$ generates a dilation in the coordinate $\chi=e^{\int d z / z \tilde{f}(z)}$.

[58] P. Calabrese and J. L. Cardy, Entanglement Entropy and Quantum Field Theory, J. Stat. Mech. (2004) P06002.

[59] P. Calabrese and J. L. Cardy, Evolution of Entanglement Entropy in One-Dimensional Systems, J. Stat. Mech. (2005) P04010.

[60] The conformal boundary condition ensures that the magnitude of the velocity remains the same after bouncing.

[61] More exactly, this statement is true only for a vanishing function at least faster than linear, because $t \sim \int_{0}^{\epsilon}\left(d x / x^{a}\right)$ diverges when $a \geq 1$. Here, for the SSD, we have $v(x)=$ $2 \sin ^{2}(\pi x / L) 2 \sim x^{2}$ for $x \rightarrow 0$.

[62] B. Chen and J.-q. Wu, Holographic Entanglement Entropy for a Large Class of States in 2D CFT, J. High Energy Phys. 09 (2016) 015. 
[63] Based on Eqs. (B2) and (B3), one can find the difference of $m$ th Renyi entropy as follows:

$$
\begin{aligned}
& S_{A}^{(m)}(n)-S_{A}^{(m)}(0) \\
&=\frac{1}{1-m} \log \left[\left(\frac{\partial z_{n}}{\partial z}\right)^{h_{m}} \cdot\left(\frac{\partial \bar{z}_{n}}{\partial \bar{z}}\right)^{h_{m}} \cdot \frac{\mathcal{T}_{m}\left(z_{n}, \bar{z}_{n}\right)}{\mathcal{T}_{m}(z, \bar{z})}\right] \\
&= \frac{1}{1-m} \log \left[\left(\frac{\partial z_{n}}{\partial z}\right)^{h_{m}}\left(\frac{\partial \bar{z}_{n}}{\partial \bar{z}}\right)^{h_{m}} .\right. \\
&\left.\times\left(\frac{\sqrt{z} \cdot \sqrt{\bar{z}}}{\sqrt{z_{n}} \sqrt{\bar{z}_{n}}}\right)^{h_{m}}\left(\frac{\sqrt{z}-\sqrt{\bar{z}}}{\sqrt{z_{n}}-\sqrt{\bar{z}_{n}}}\right)^{2 h_{m}}\right],
\end{aligned}
$$

where $\partial z_{n} / \partial z$ and $\partial \bar{z}_{n} / \partial \bar{z}$ are calculated through the chain rule in Eq. (44). $z=e^{2 \pi i(l / L)}$ depends only on the ratio $l / L$. $z_{n}\left(\bar{z}_{n}\right)$ are determined by Eqs. (44) and (45), which depend only on the dimensionless parameters $l / L, T_{0} / L$, and $T_{1} / L$ in Eq. (41). It is noted that $T_{0}$ and $T_{1}$ in Eq. (41) are random numbers. After doing an average over Eq. (46), the result depends only on $l / L, \bar{T}_{0} / L, \bar{T}_{1} / L$, and $\alpha$.

[64] Just to remind that the reason we choose an open boundary condition for the $q=1$ case is that, in the periodic boundary condition, the ground state of $H_{0}$ is annihilated by $H_{1}$ as well as due to the SL(2) symmetry.

[65] Although the algebra is isomorphic, we may emphasize the different group action by denoting the subgroup as $\operatorname{SL}^{(m)}(2, \mathbb{R})$, which represents an $m$-fold cover of $\operatorname{SL}(2, \mathbb{R})$.

[66] The prefactor differs by $\frac{4}{3}\left(q^{2}-1\right)$, where $\frac{4}{3}$ is due to the shift of the boundary condition from open to periodic in this section.

[67] I. Bloch, Ultracold Quantum Gases in Optical Lattices, Nat. Phys. 1, 23 (2005).

[68] R. Islam, R. Ma, P. M. Preiss, M. E. Tai, A. Lukin, M. Rispoli, and M. Greiner, Measuring Entanglement Entropy in a Quantum Many-Body System, Nature (London) 528, 77 (2015).

[69] The intuitive reason is that in the heating phase both $z_{n}$ and $\bar{z}_{n}$ flow to the attractive fixed point $\gamma_{1}$ as an exponential function of $n$. Here, we assume that neither $z_{n}$ nor $\bar{z}_{n}$ collides with the repulsive fixed point.

[70] This result is consistent with the convention that, at the imaginary time, when going to the upper half plane geometry, $\xi$ has to sit on the upper half plane, while $\bar{\xi}$ has to sit on the lower half plane. 